\title{
The Relay-Eavesdropper Channel: Cooperation for Secrecy
}

\author{
Lifeng Lai and Hesham El Gamal
}

\begin{abstract}
This paper establishes the utility of user cooperation in facilitating secure wireless communications. In particular, the four-terminal relay-eavesdropper channel is introduced and an outer-bound on the optimal rate-equivocation region is derived. Several cooperation strategies are then devised and the corresponding achievable rate-equivocation region are characterized. Of particular interest is the novel Noise-Forwarding (NF) strategy, where the relay node sends codewords independent of the source message to confuse the eavesdropper. This strategy is used to illustrate the deaf helper phenomenon, where the relay is able to facilitate secure communications while being totally ignorant of the transmitted messages. Furthermore, NF is shown to increase the secrecy capacity in the reversely degraded scenario, where the relay node fails to offer performance gains in the classical setting. The gain offered by the proposed cooperation strategies is then proved theoretically and validated numerically in the additive White Gaussian Noise (AWGN) channel.
\end{abstract}

\section{INTRODUCTION}

Shannon introduced the notion of information theoretic secrecy in [1]. The model in [1] assumed that the transmission is noiseless, and used a key $K$ to protect the confidential message $W$. Taking the transmission uncertainty into consideration, Wyner introduced the wiretap channel in [2]. In the three-terminal wiretap channel, a source wishes to transmit confidential messages to a destination while keeping the messages as secret as possible from a wiretapper. The wiretapper is assumed to have an unlimited computation ability and to know the coding/decoding scheme used in the main (source-destination) channel. Under the assumption that the source-wiretapper channel is a degraded version of the main channel, Wyner characterized the trade-off between

The authors are with the ECE Department at the Ohio State University. Email:\{lail,helgamal\}@ece.osu.edu. 
the throughput of the main channel and the level of ignorance of the message at the wiretapper using the rate-equivocation region concept. Loosely speaking, the equivocation rate measures the residual ambiguity about the transmitted message at the wiretapper. If the equivocation rate at the wiretapper is arbitrarily close to the information rate, the transmission is called perfectly secure. Csiszár and Körner extended this work to the broadcast channel with confidential messages, where the source sends common information to both the destination and the wiretapper, and confidential messages are sent only to the destination [3].

Our work here is motivated by the fact that if the wiretapper channel is less noisy than the main channel1, the perfect secrecy capacity of the channel is zero [3]. In this case, it is infeasible to establish a secure link under Wyner's wiretap channel model. Our main idea is to exploit user cooperation in facilitating the transmission of confidential messages from the source to the destination. More specially, we consider a four-terminal relay-eavesdropper channel, where a source wishes to send messages to a destination while leveraging the help of a relay node to hide those messages from the eavesdropper. The eavesdropper in our model can be viewed as the wireless counterpart of Wyner's wiretapper. This model generalizes the relay channel [4] and the wiretap channel [2].

The relay channel without security constraints was studied under various scenarios [4]-[12]. In most of these works, cooperation strategies were constructed to increase the transmission rate and/or reliability function. In this paper, we identify a novel role of the relay node in establishing a secure link from the source to the destination. Towards this end, several cooperation strategies for the relay-eavesdropper channel are constructed and the corresponding achieved rateequivocation regions are characterized. An outer-bound on the optimal rate-equivocation region is also derived. The proposed schemes are shown to achieve a positive perfect secrecy rate in several scenarios where the secrecy capacity in the absence of the relay node is zero. Quite interestingly, we establish the deaf-helper phenomenon where the relay can help while being totally ignorant of the transmitted message from the source. Furthermore, we show that the relay node can aid in the transmission of confidential messages in some settings where classical cooperation fails to offer performance gains, e.g., the reversely degraded relay channel. Finally,

\footnotetext{
${ }^{1}$ The source-wiretapper channel is said to be less noisy than the source-receiver channel, if for every $V \rightarrow X \rightarrow Y Z$, $I(V ; Z) \geq I(V ; Y)$, where $X$ is the signal transmitted by the source, $Y, Z$ are the received signal of the receiver and the wiretapper respectively.
} 
we observe that the proposed Noise-Forwarding (NF) is intimately related with the multiple access channel with security constraints, as evident in the sequel.

At this point, we wish to differentiate our investigation from earlier relevant works. The relay channel with confidential messages was studied in [13], [14], where the relay node acts both as an eavesdropper and a helper. In the model of [14], the source sends common messages to the destination using the help of the relay node, but also sends private messages to the destination while keeping them secret from the relay. In contrast with [14], the relay node in our work acts as a trusted "third-party" whose sole goal is to facilitate secure communications (imposing an additional security constraint on the relay node is also considered in Section IV]. The idea of using a "third-party" to facilitate secure communications also appeared in [15]. Contrary to our work, which considers noisy channels, [15] focused on the generation of common random secret keys at two nodes under the assist of a third-party using a noiseless public discussion channel. The users then use the secret key to establish a secure link between the source-destination pair. Other recent works on secure communications investigated the multiple access channel (MAC) with confidential messages [16], [17], the multiple access channel with a degraded wiretapper [18], and MIMO secure communications [19]. In summary, it appears that our relay-eavesdropper model is fundamentally different from the models considered in all previous works.

Throughout the paper, upper-case letter $X$ denotes a random variable, lower-case letter $x$ denotes a realization of the random variable, calligraphic letter $\mathcal{X}$ denotes a finite alphabet set. Boldface letter $\mathrm{x}$ denotes a vector, $\{\cdot\}^{T}$ denotes transpose and $\{\cdot\}^{H}$ denotes conjugate transpose. We also let $[x]^{+}=\max \{0, x\}$.

The rest of the paper is organized as follows. In section II, we introduce the system model and our notations. Section III describes the proposed cooperation strategies and characterizes the corresponding achievable performance. The rate-equivocation outer-bound is also developed in this section. In Section IV] we discuss several examples that illustrate interesting aspects of the relay-eavesdropper channel. Finally, Section $\nabla]$ offers some concluding remarks and briefly outlines possible venues for future research.

\section{The RELAY-EAVESDROPPER CHANNEL}

We consider a four-terminal discrete channel consisting of finite sets $\mathcal{X}_{1}, \mathcal{X}_{2}, \mathcal{Y}, \mathcal{Y}_{1}, \mathcal{Y}_{2}$ and a transition probability distribution $p\left(y, y_{1}, y_{2} \mid x_{1}, x_{2}\right)$, as shown in Figure 1 . Here, $\mathcal{X}_{1}, \mathcal{X}_{2}$ are the 
channel inputs from the source and the relay respectively, while $\mathcal{Y}, \mathcal{Y}_{1}, \mathcal{Y}_{2}$ are the channel outputs at the destination, relay and eavesdropper respectively. We impose the memoryless assumption, $i$.e., the channel outputs $\left(y_{i}, y_{1, i}, y_{2, i}\right)$ at time $i$ only depend on the channel inputs $\left(x_{1, i}, x_{2, i}\right)$ at time $i$. The source wishes to send the message $W_{1} \in \mathcal{W}_{1}=\{1, \cdots, M\}$ to the destination using the $(M, n)$ code consisting: 1$)$ a stochastic encoder $f_{n}$ at the source that maps the message $w_{1}$ to a codeword $\left.\mathbf{x}_{1} \in \mathcal{X}_{1}^{n}, 2\right)$ a relay encoder that maps the signals $\left(y_{1,1}, y_{1,2}, \cdots, y_{1, i-1}\right)$ received before time $i$ to the channel input $x_{2, i}$, using the mapping $\left.\varphi_{i}:\left(Y_{1,1}, Y_{1,2}, \cdots, Y_{1, i-1}\right) \rightarrow X_{2, i}, 3\right)$ a decoding function $\phi: \mathcal{Y}^{n} \rightarrow \mathcal{W}_{1}$. The average error probability of a $(M, n)$ code is defined as

$$
P_{e}^{n}=\sum_{w_{1} \in \mathcal{W}_{1}} \frac{1}{M} \operatorname{Pr}\left\{\phi(\mathbf{y}) \neq w_{1} \mid w_{1} \text { was sent }\right\} .
$$

The equivocation rate at the eavesdropper is defined as

$$
R_{e}=\frac{1}{n} H\left(W_{1} \mid \mathbf{Y}_{2}\right) .
$$

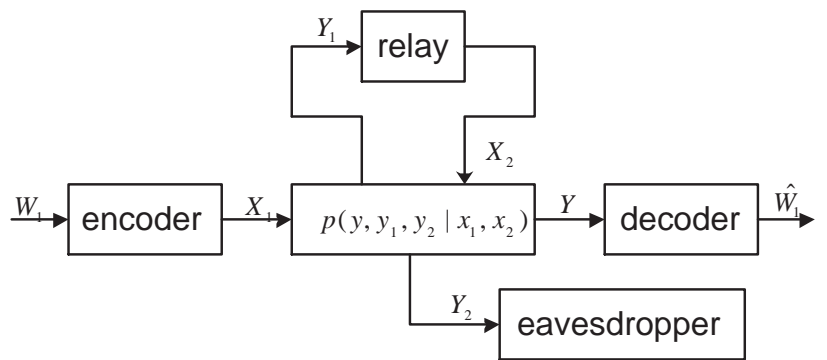

Fig. 1: The relay eavesdropper channel.

The rate-equivocation pair $\left(R_{1}, R_{e}\right)$ is said to be achievable if for any $\epsilon>0$, there exists a sequence of codes $(M, n)$ such that for any $n \geq n(\epsilon)$, we have

$$
\begin{aligned}
R_{1} & =\frac{1}{n} \log _{2} M, \\
P_{e}^{n} & \leq \epsilon, \\
\frac{1}{n} H\left(W_{1} \mid \mathbf{Y}_{2}\right) & \geq R_{e}-\epsilon .
\end{aligned}
$$

We further say that the perfect secrecy rate $R_{1}$ is achievable if the rate-equivocation pair $\left(R_{1}, R_{1}\right)$ is achievable. Notice that if $Y_{2}=\phi$ (or some other constant), our model reduces to the classical relay channel without security constraints. 


\section{MAIN RESULTS}

Our first result establishes an outer-bound on the optimal rate-equivocation region of the relay-eavesdropper channel.

Theorem 1: In the relay eavesdropper channel, for any rate-equivocation pair $\left\{R_{1}, R_{e}\right\}$ with $P_{e}^{n} \rightarrow 0$ and the equivocation rate at the eavesdropper larger than $R_{e}-\epsilon$, there exist some random variables $U \rightarrow\left(V_{1}, V_{2}\right) \rightarrow\left(X_{1}, X_{2}\right) \rightarrow\left(Y, Y_{1}, Y_{2}\right)$, such that $\left(R_{1}, R_{e}\right)$ satisfies the following conditions

$$
\begin{aligned}
& R_{1} \leq \min \left\{I\left(V_{1}, V_{2} ; Y\right), I\left(V_{1} ; Y, Y_{1} \mid V_{2}\right)\right\} \\
& R_{e} \leq R_{1}, \\
& R_{e} \leq\left[I\left(V_{1}, V_{2} ; Y \mid U\right)-I\left(V_{1}, V_{2} ; Y_{2} \mid U\right)\right]^{+} .
\end{aligned}
$$

Proof: Please refer to Appendix I]

We now turn our attention to constructing cooperation strategies for the relay-eavesdropper channel. Our first step is to characterize the achievable rate-equivocation region of Cover-El Gamal Decode and Forward (DF) Strategy [4]. In DF cooperation strategy, the relay node will first decode codewords and then re-encode the message to cooperate with the source. Here, we use the regular coding and backward decoding scheme developed in the classical relay setting [7], [20], with the important difference that each message will be associated with many codewords in order to confuse the eavesdropper.

Theorem 2: The rate pairs in the closure of the convex hull of all $\left(R_{1}, R_{e}\right)$ satisfying

$$
\begin{aligned}
& R_{1}<\min \left\{I\left(V_{1}, V_{2} ; Y\right), I\left(V_{1} ; Y_{1} \mid V_{2}\right)\right\}, \\
& R_{e}<R_{1}, \\
& R_{e}<\left[\min \left\{I\left(V_{1}, V_{2} ; Y\right), I\left(V_{1} ; Y_{1} \mid V_{2}\right)\right\}-I\left(V_{1}, V_{2} ; Y_{2}\right)\right]^{+},
\end{aligned}
$$

for some distribution $p\left(v_{1}, v_{2}, x_{1}, x_{2}, y_{1}, y_{2}, y\right)=p\left(v_{1}, v_{2}\right) p\left(x_{1}, x_{2} \mid v_{1}, v_{2}\right) p\left(y_{1}, y_{2}, y \mid x_{1}, x_{2}\right)$, are achievable using the DF strategy.

Hence, for the DF scheme, the following perfect secrecy rate is achievable

$$
R_{s}^{(D F)}=\sup _{p\left(v_{1}, v_{2}\right)}\left[\min \left\{I\left(V_{1}, V_{2} ; Y\right), I\left(V_{1} ; Y_{1} \mid V_{2}\right)\right\}-I\left(V_{1}, V_{2} ; Y_{2}\right)\right]^{+} .
$$

Proof: Please refer to Appendix [II. 
The channel between the source and the relay becomes a bottleneck for the DF strategy when it is noisier than the source-destination channel. This motivates our Noise-Forwarding (NF) scheme, where the relay node does not attempt to decode the message but sends codewords that are independent of the source's message. The enabling observation behind this scheme is that, in the wiretap channel, in addition to its own information, the source should send extra codewords to confuse the wiretapper. In our setting, this task can be accomplished by the relay by allowing it to send independent codewords, which aid in confusing the eavesdropper.
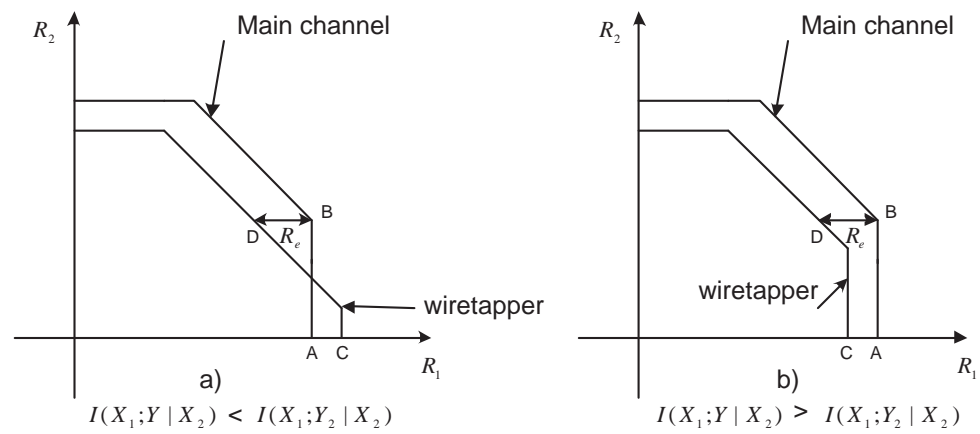

Fig. 2: The rate region of the compound MACs of the relay eavesdropper channel for a fixed input distribution $p\left(x_{1}\right) p\left(x_{2}\right)$.

Our NF scheme transforms the relay-eavesdropper channel into a compound multiple access channel (MAC), where the source/relay to the receiver is the first MAC and source/relay to the eavesdropper is the second one. Figure 2 shows the rate region of these two MACs for a fixed input distribution $p\left(x_{1}\right) p\left(x_{2}\right)$. In the figure, $R_{1}$ is the codeword rate of the source, and $R_{2}$ is the codeword rate of the relay. We can observe from Figure 2a) that if the relay node does not transmit, the perfect secrecy rate is zero for this input distribution since $R_{1}(A)<R_{1}(C)$. On the other hand, if the relay and the source coordinate their transmissions and operate at point $B$, we can achieve the equivocation rate $R_{e}$, which is strictly larger than zero. On the other hand, in Figure $2 b$ ), we can still get a positive perfect secrecy rate by operating at point $A$ in the absence of the relay. But by moving the operating point to $B$, we can get a larger secrecy rate. This illustrates the main idea of our Noise-Forwarding scheme. The next result establishes the achievable rate-equivocation region for the NF scheme. 
Theorem 3: The rate pairs in the closure of the convex hull of all $\left(R_{1}, R_{e}\right)$ satisfying

$R_{1}<I\left(V_{1} ; Y \mid V_{2}\right)$

$R_{e}<R_{1}$

$R_{e}<\left[I\left(V_{1} ; Y \mid V_{2}\right)+\min \left\{I\left(V_{2} ; Y\right), I\left(V_{2} ; Y_{2} \mid V_{1}\right)\right\}-\min \left\{I\left(V_{2} ; Y\right), I\left(V_{2} ; Y_{2}\right)\right\}-I\left(V_{1} ; Y_{2} \mid V_{2}\right)\right]^{+}$,

for some distribution $p\left(v_{1}, v_{2}, x_{1}, x_{2}, y_{1}, y_{2}, y\right)=p\left(v_{1}\right) p\left(v_{2}\right) p\left(x_{1} \mid v_{1}\right) p\left(x_{2} \mid v_{2}\right) p\left(y_{1}, y_{2}, y \mid x_{1}, x_{2}\right)$, are achievable using the NF scheme.

Hence, for the NF scheme, the achievable perfect secrecy rate is

$$
\begin{aligned}
R_{s}^{(N F)=} \sup _{p\left(v_{1}\right) p\left(v_{2}\right)}[ & I\left(V_{1} ; Y \mid V_{2}\right)+\min \left\{I\left(V_{2} ; Y\right), I\left(V_{2} ; Y_{2} \mid V_{1}\right)\right\} \\
& \left.-\min \left\{I\left(V_{2} ; Y\right), I\left(V_{2} ; Y_{2}\right)\right\}-I\left(V_{1} ; Y_{2} \mid V_{2}\right)\right]^{+} .
\end{aligned}
$$

Proof: Please refer to Appendix III

The following comments are now in order.

1) The NF scheme is customized to the relay channel with security constraints which make the transmission of codewords that are independent of the source message reasonable. Also, in the NF scheme, the relay node does not need to listen to the source, and hence, this scheme is more suited works for relay nodes limited by the half-duplex constraint [9], [11], [21].

2) In NF cooperation, each user sends independent messages to the destination, which resembles the MAC. Hence, NF cooperation can be adapted to the multiple access eavesdropper channel where the multiple users in the MAC channel can help each other in communicating securely with the destination without listening to each other (note that the results in [18] were limited only to the case where the eavesdropped channel is a degraded version of the channel seen by the destination). Our related results will be reported elsewhere.

Now, we study another cooperation scheme that does not require decoding at the relay: Compress and Forward (CF). The CF cooperation strategy can be viewed as a generalization of $\mathrm{NF}$ where, in addition to the independent codewords, the relay also sends a quantized version of its noisy observations to the destination. This noisy version of the relay's observations helps the destination in decoding the source's message, while the independent codewords help in confusing the eavesdropper. The following result establishes the achievable rate-equivocation pair in the 
case when $I\left(X_{1} ; \hat{Y}_{1}, Y \mid X_{2}\right) \leq I\left(X_{1} ; \hat{Y}_{1}, Y_{2} \mid X_{2}\right)$, i.e., the source-eavesdropper channel is better than the source-receiver channel, a situation of particular interest to us.

Theorem 4: The rate pairs in the closure of the convex hull of all $\left(R_{1}, R_{e}\right)$ satisfying

$$
\begin{aligned}
& R_{1}<I\left(X_{1} ; \hat{Y}_{1}, Y \mid X_{2}\right), \\
& R_{e}<R_{1}, \\
& R_{e}<\left[R_{0}+I\left(X_{1} ; \hat{Y}_{1}, Y \mid X_{2}\right)-I\left(X_{1}, X_{2} ; Y_{2}\right)\right]^{+},
\end{aligned}
$$

subject to

$$
\min \left\{I\left(X_{2} ; Y\right), I\left(X_{2} ; Y_{2} \mid X_{1}\right)\right\}-R_{0} \geq I\left(Y_{1} ; \hat{Y}_{1} \mid X_{2}\right),
$$

for some distribution $p\left(x_{1}, x_{2}, y_{1}, y_{2}, y, \hat{y}_{1}\right)=p\left(x_{1}\right) p\left(x_{2}\right) p\left(y_{1}, y_{2}, y \mid x_{1}, x_{2}\right) p\left(\hat{y}_{1} \mid y_{1}, x_{2}\right)$, are achievable using CF strategy.

Proof: Please refer to Appendix IV.

Three comments are now in order.

1) In Theorem $4, R_{0}$ is the rate of pure noise generated by the relay to confuse the eavesdropper, while $\min \left\{I\left(X_{2} ; Y\right), I\left(X_{2} ; Y_{2} \mid X_{1}\right)\right\}-R_{0}$ is the part of the rate allocated to send the compressed signal $\hat{Y}_{1}$ to help the destination. If we set $R_{0}=\min \left\{I\left(X_{2} ; Y\right), I\left(X_{2} ; Y_{2} \mid X_{1}\right)\right\}$, this scheme becomes the NF scheme.

2) In order to enable analytical tractability, the coding/decoding scheme used in the proof is slightly different from that of [4]. In [4], the destination uses sliding-window decoding, while our proof uses backward decoding. Hence, the bound for $R_{e}$ provided here is a lower-bound for the $R_{e}$ achieved by the CF scheme. One may be able to achieve a larger $R_{e}$ using exactly the CF scheme proposed in [4]. But, unfortunately, we are not yet able to bound $R_{e}$ when sliding-window decoding is used.

3) Compared with CF decoding, the proposed NF strategy enjoys the advantage of simplicity. Also, if one only focuses on the perfect secrecy rate, it is easy to see that these two schemes achieve identical performance. Again, this observation is limited to our lower bound on $R_{e}$ in Theorem 4

\section{EXAMPLES}

This section discusses several examples that illustrate some unique features of the relayeavesdropper channel. For simplicity, we only focus on the perfect secrecy rate of various 
schemes.

\section{A. The Deaf Helper Phenomenon}

The security constraints imposed on the network bring about a new phenomenon which we call the deaf helper phenomenon, where the relay node can still help even it is totally ignorant of the message transmitted from the source. In this setup, we impose an additional security constraint on the relay node, and say a rate $R_{s}$ is achievable for a deaf helper if for any $\epsilon>0$, there exists a sequence of codes $(M, n)$ such that for any $n \geq n(\epsilon)$, we have

$$
\begin{aligned}
R_{s} & =\frac{1}{n} \log _{2} M, \quad P_{e}^{n} \leq \epsilon, \\
\frac{1}{n} H\left(W_{1} \mid \mathbf{Y}_{2}\right) & \geq R_{s}-\epsilon, \quad \frac{1}{n} H\left(W_{1} \mid \mathbf{Y}_{1}, \mathbf{X}_{2}\right) \geq R_{s}-\epsilon .
\end{aligned}
$$

In this case, the signal received by the relay node does not leak any information about the transmitted message $W_{1}$. This model describes a more conservative scenario where the source does not trust the relay but still wishes to exploit the benefit brought by cooperation. We assume that the relay node is not malicious and, hence, is willing to cooperate with the source 2 . The following theorem characterizes the achievable perfect secrecy rate of the NF strategy in the deaf-helper setting.

Theorem 5: The perfect secrecy rate of the NF scheme with an additional security constraint on the relay node is $R_{s}=\max _{p\left(v_{1}\right) p\left(v_{2}\right)} \min \left\{R_{s 1}, R_{s 2}\right\}$, where

$$
\begin{aligned}
& R_{s 1}=\left[I\left(V_{1} ; Y \mid V_{2}\right)+\min \left\{I\left(V_{2} ; Y\right), I\left(V_{2} ; Y_{2} \mid V_{1}\right)\right\}-\min \left\{I\left(V_{2} ; Y\right), I\left(V_{2} ; Y_{2}\right)\right\}-I\left(V_{1} ; Y_{2} \mid V_{2}\right)\right]^{+}, \\
& R_{s 2}=\left[I\left(V_{1} ; Y \mid V_{2}\right)-I\left(V_{1} ; Y_{1} \mid X_{2}\right)\right]^{+} .
\end{aligned}
$$

Proof: Please refer to Appendix V.

\section{B. The Reversely Degraded Relay-Eavesdropper Channel}

In the classical relay channel without security constraints, there exist some scenarios where the relay node does not provide any gain, for example, the reversely degraded relay channel shown in [4]. Here, we focus on this scenario and show that the relay node can still offer a gain in the presence of the eavesdropper.

\footnotetext{
${ }^{2}$ If the relay node is malicious, it can then send signals that are dependent with signal received and then could even block the transmission of the main channel.
} 
Definition 1 ( [4]): The relay channel is called reversely degraded, if $p\left(y, y_{1} \mid x_{1}, x_{2}\right)=p\left(y \mid x_{1}, x_{2}\right)$ $p\left(y_{1} \mid y, x_{2}\right)$.

The following result, borrowed from [4], states the capacity of the classical reversely degraded relay channel.

Theorem 6 (Theorem 2, [4]): The capacity of the reversely degraded relay channel is

$$
C_{0}=\max _{x_{2}} \max _{p\left(x_{1}\right)} I\left(X_{1} ; Y \mid x_{2}\right) .
$$

This result implies that the relay node should send a constant, and hence, does not contribute new information to the destination. In most channel models, the constant sent by the relay does not result in any capacity gain. The question now is whether the same conclusion holds in the presence of an eavesdropper. We first observe that the degradedness of the relay channel implies that DF and CF cooperation will not provide the destination with additional useful information. The relay node, however, can still send codewords independent of the received signal to confuse the eavesdropper, as proposed in the NF scheme. Since we do not require decoding at the relay node in the proof of Theorem 3, the degradedness imposed here does not affect the performance. Hence, we get the following achievable perfect secrecy rate for the reversely degraded relayeavesdropper channel.

Corollary 1: The achievable perfect secrecy rate of the reversely degraded relay eavesdropper channel is

$$
\begin{aligned}
R_{s}=\max _{p\left(v_{1}\right) p\left(v_{2}\right)}[ & I\left(V_{1} ; Y \mid V_{2}\right)+\min \left\{I\left(V_{2} ; Y\right), I\left(V_{2} ; Y_{2} \mid V_{1}\right)\right\} \\
& \left.-\min \left\{I\left(V_{2} ; Y\right), I\left(V_{2} ; Y_{2}\right)\right\}-I\left(V_{1} ; Y_{2} \mid V_{2}\right)\right]^{+} .
\end{aligned}
$$

\section{The AWGN Channel}

Now we consider the Gaussian relay-eavesdropper channel, where the signal received at each node is

$$
y_{j}[n]=\sum_{i \neq j} h_{i j} x_{i}[n]+z_{j}[n],
$$

here $h_{i j}$ is the channel coefficient between node $i \in\{s, r\}$ and node $j \in\{r, w, d\}$, and $z_{j}$ is the i.i.d Gaussian noise with unit variance at node $j$. The source and the relay have average power constraint $P_{1}, P_{2}$ respectively. 
In [22], it was shown that the secrecy capacity of the degraded Gaussian wiretap channel is $\left[C_{M}-C_{M W}\right]^{+}$, where $C_{M}, C_{M W}$ are the capacity of the main channel and wiretap channel, respectively. This result is also shown to be valid for stochastically degraded channel [17]. In our case, if the relay does not transmit, the relay eavesdropper channel becomes a Gaussian eavesdropper channel, which can always be converted into a stochastically degraded channel as done in the Gaussian broadcast channel [23]. Applying this result to our case, the secrecy capacity of the Gaussian eavesdropper channel without the relay node is given by $\left[\frac{1}{2} \log _{2}\left(1+\left|h_{s d}\right|^{2} P_{1}\right)-\frac{1}{2} \log _{2}\left(1+\left|h_{s w}\right|^{2} P_{1}\right)\right]^{+}$. Hence if $\left|h_{s w}\right|^{2} \geq\left|h_{s d}\right|^{2}$ and the relay does not transmit, the secrecy capacity is zero, no matter how large $P_{1}$ is. On the other hand, as shown later, the relay can facilitate the source-destination pair to achieve a positive perfect secrecy rate under some conditions even when $\left|h_{s w}\right|^{2} \geq\left|h_{s d}\right|^{2}$. In the following, we focus on such scenarios.

1) $D F$ and NF: At this point, we do not know the optimal input distribution that maximizes $R_{s}^{(D F)}, R_{s}^{(N F)}$. Here, we let $V_{1}=X_{1}, V_{2}=X_{2}$ and use a Gaussian input distribution to obtain an achievable lower bound.

For DF cooperation scheme, we let $X_{2} \sim \mathcal{N}\left(0, P_{2}\right), X_{10} \sim \mathcal{N}(0, P)$, where $\mathcal{N}(0, P)$ is the Gaussian distribution with zero mean and variance $P$. Also, we let

$$
X_{1}=c_{1} X_{2}+X_{10}
$$

where $c_{1}$ is a constant to be specified later. In this relationship, the novel information is modelled by $X_{10}$, whereas $X_{2}$ represents the part of the signal which the source and the relay cooperate in beamforming towards the destination. To satisfy the average power constraint at the source, we require $\left|c_{1}\right|^{2} P_{2}+P \leq P_{1}$.

Straightforward calculations result in

$$
\begin{aligned}
I\left(X_{1} ; Y_{1} \mid X_{2}\right) & =\frac{1}{2} \log _{2}\left(1+\left|h_{s r}\right|^{2} P\right) \\
I\left(X_{1}, X_{2} ; Y\right) & =\frac{1}{2} \log _{2}\left(1+\left|h_{s d} c_{1}+h_{r d}\right|^{2} P_{2}+\left|h_{s d}\right|^{2} P\right) \\
I\left(X_{1}, X_{2} ; Y_{2}\right) & =\frac{1}{2} \log _{2}\left(1+\left|h_{s w} c_{1}+h_{r w}\right|^{2} P_{2}+\left|h_{s w}\right|^{2} P\right)
\end{aligned}
$$

Hence, we have

$$
\begin{array}{r}
R_{s}^{(D F)}=\max _{c_{1}, P}\left[\operatorname { m i n } \left\{\frac{1}{2} \log _{2}\left(\frac{1+\left|h_{s r}\right|^{2} P}{1+\left|h_{s w} c_{1}+h_{r w}\right|^{2} P_{2}+\left|h_{s w}\right|^{2} P}\right),\right.\right. \\
\left.\left.\quad \frac{1}{2} \log _{2}\left(\frac{1+\left|h_{s d} c_{1}+h_{r d}\right|^{2} P_{2}+\left|h_{s d}\right|^{2} P}{1+\left|h_{s w} c_{1}+h_{r w}\right|^{2} P_{2}+\left|h_{s w}\right|^{2} P}\right)\right\}\right]^{+} .
\end{array}
$$


For NF, we let $X_{1} \sim \mathcal{N}\left(0, P_{1}\right), X_{2} \sim \mathcal{N}\left(0, P_{2}\right)$. Here $X_{1}, X_{2}$ are independent, resulting in

$$
\begin{aligned}
& I\left(X_{1} ; Y \mid X_{2}\right)=\frac{1}{2} \log _{2}\left(1+\left|h_{s d}\right|^{2} P_{1}\right), \\
& I\left(X_{1}, X_{2} ; Y\right)-I\left(X_{1}, X_{2} ; Y_{2}\right)=\frac{1}{2} \log _{2}\left(\frac{1+\left|h_{s d}\right|^{2} P_{1}+\left|h_{r d}\right|^{2} P_{2}}{1+\left|h_{s w}\right|^{2} P_{1}+\left|h_{r w}\right|^{2} P_{2}}\right), \\
& I\left(X_{2} ; Y_{2} \mid X_{1}\right)+I\left(X_{1} ; Y \mid X_{2}\right)-I\left(X_{1}, X_{2} ; Y_{2}\right)=\frac{1}{2} \log _{2}\left(\frac{\left(1+\left|h_{r w}\right|^{2} P_{2}\right)\left(1+\left|h_{s d}\right|^{2} P_{1}\right)}{1+\left|h_{s w}\right|^{2} P_{1}+\left|h_{r w}\right|^{2} P_{2}}\right) .
\end{aligned}
$$

Hence, we have

$$
\begin{aligned}
R_{s}^{(N F)=}\left[\operatorname { m i n } \left\{\frac{1}{2} \log _{2}\left(1+\left|h_{s d}\right|^{2} P_{1}\right), \frac{1}{2} \log _{2}\left(\frac{1+\left|h_{s d}\right|^{2} P_{1}+\left|h_{r d}\right|^{2} P_{2}}{1+\left|h_{s w}\right|^{2} P_{1}+\left|h_{r w}\right|^{2} P_{2}}\right),\right.\right. \\
\\
\left.\left.\frac{1}{2} \log _{2}\left(\frac{\left(1+\left|h_{r w}\right|^{2} P_{2}\right)\left(1+\left|h_{s d}\right|^{2} P_{1}\right)}{1+\left|h_{s w}\right|^{2} P_{1}+\left|h_{r w}\right|^{2} P_{2}}\right)\right\}\right]^{+} .
\end{aligned}
$$

2) Amplify and Forward: In this subsection, we quantify the achievable secrecy rate of Amplify and Forward (AF) cooperation 3 . In AF, the source encodes its messages into codewords with length $M L$ each, and divides each codeword into $L$ sub-blocks each with $M$ symbols, where $L$ is chosen to be sufficiently large. At each sub-block, the relay sends a linear combination of the received noisy signal of this sub-block so far. For simplicity, we limit our discussion to $M=2$. In this case, the source sends $X_{1}(1)$ at the first symbol interval of each sub-block, the relay receives $Y_{1}(1)=h_{s r} X_{1}(1)+Z_{1}(1)$; At the second symbol interval, the source sends $\alpha X_{1}(1)+\beta X_{1}(2)$, while the relay sends $\gamma Y_{1}(1)$. Here $\alpha, \beta, \gamma$ are chosen to satisfy the average power constraints of the source and the relay. Thus, this scheme allows beam-forming between the source and relay without requiring the relay to fully decode.

Writing the signal received at the destination and the eavesdropper in matrix form, we have

$$
\mathbf{Y}=\mathbf{H}_{1} \mathbf{X}_{1}+\mathbf{Z}, \quad \mathbf{Y}_{2}=\mathbf{H}_{2} \mathbf{X}_{1}+\mathbf{Z}_{2}
$$

where

$$
\begin{gathered}
\mathbf{H}_{1}=\left[\begin{array}{cc}
h_{s d} & 0 \\
\beta h_{s d}+\gamma h_{s r} h_{r d} & \alpha h_{s d}
\end{array}\right], \mathbf{H}_{2}=\left[\begin{array}{cc}
h_{s w} & 0 \\
\beta h_{s w}+\gamma h_{s r} h_{r w} & \alpha h_{s w}
\end{array}\right], \\
\mathbf{X}_{1}=\left[X_{1}(1), X_{1}(2)\right]^{T}, \mathbf{Z}=\left[Z(1), \gamma h_{r d} Z_{1}(1)+Z(2)\right]^{T}, \mathbf{Z}_{2}=\left[Z_{2}(1), \gamma h_{r w} Z_{1}(1)+Z_{2}(2)\right]^{T}, \\
\mathbf{Y}=[Y(1), Y(2)]^{T}, \mathbf{Y}_{2}=\left[Y_{2}(1), Y_{2}(2)\right]^{T} .
\end{gathered}
$$

\footnotetext{
${ }^{3}$ We did not consider this scheme in the discrete case since, in general, it does not lend itself to a single letter characterization.
} 
The channel under consideration can be viewed as an equivalent standard memoryless eavesdropper channel with input $\mathbf{X}_{1}$ and outputs $\mathbf{Y}, \mathbf{Y}_{2}$ at the destination and the eavesdropper respectively. Then, based on the result of [3], an achievable perfect secrecy rate is $\left[I\left(\mathbf{X}_{1} ; \mathbf{Y}\right)-I\left(\mathbf{X}_{1} ; \mathbf{Y}_{2}\right)\right]^{+}$.

Choosing a Gaussian input with covariance matrix $\mathbb{E}\left\{\mathbf{X X}^{H}\right\}=P \mathbf{I}$, where $\mathbf{I}$ is the identity matrix, we get the following perfect secrecy rate

$$
\begin{aligned}
R_{s}^{(A F)} & =\max _{\alpha, \beta, \gamma, P}\left[\frac{1}{4} \log _{2} \frac{\left|\operatorname{det}\left\{P \mathbf{H}_{1} \mathbf{H}_{1}^{H}+\mathbb{E}\left\{\mathbf{Z} \mathbf{Z}^{H}\right\}\right\}\right|}{\left|\operatorname{det}\left\{\mathbb{E}\left\{\mathbf{Z} \mathbf{Z}^{H}\right\}\right\}\right|}-\frac{1}{4} \log _{2} \frac{\left|\operatorname{det}\left\{P \mathbf{H}_{2} \mathbf{H}_{2}^{H}+\mathbb{E}\left\{\mathbf{Z}_{2} \mathbf{Z}_{2}^{H}\right\}\right\}\right|}{\left|\operatorname{det}\left\{\mathbb{E}\left\{\mathbf{Z}_{2} \mathbf{Z}_{2}^{H}\right\}\right\}\right|}\right]^{+} \\
& =\max _{\alpha, \beta, \gamma, P}\left[\frac{1}{4} \log _{2} \frac{\left|\operatorname{det}\left\{P \mathbf{H}_{1} \mathbf{H}_{1}^{H}+\mathbf{A}\right\} \operatorname{det} \mathbf{B}\right|}{\left|\operatorname{det}\left\{P \mathbf{H}_{2} \mathbf{H}_{2}^{H}+\mathbf{B}\right\} \operatorname{det} \mathbf{A}\right|}\right]^{+}
\end{aligned}
$$

where

$$
\mathbf{A}=\left[\begin{array}{cc}
1 & 0 \\
0 & 1+\left|\gamma h_{r d}\right|^{2}
\end{array}\right], \mathbf{B}=\left[\begin{array}{cc}
1 & 0 \\
0 & 1+\left|\gamma h_{r w}\right|^{2}
\end{array}\right],
$$

and the maximization is over the set of power constraints:

$$
\begin{gathered}
\left(1+|\alpha|^{2}+|\beta|^{2}\right) P \leq 2 P_{1}, \\
|\gamma|^{2}\left(\left|h_{s r}\right|^{2} P+1\right) \leq 2 P_{2} .
\end{gathered}
$$

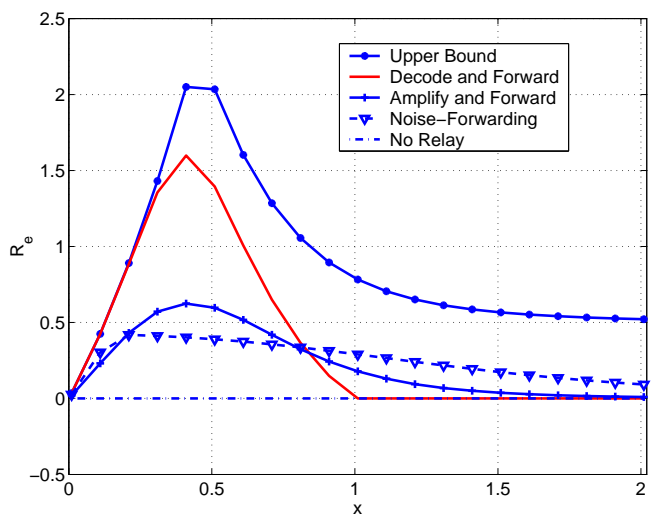

Fig. 3: The achievable perfect secrecy rate of the proposed schemes in the Gaussian relay eavesdropper channel.

3) Numerical Results: In this subsection, we give numerical results under two channel models. The first is the real channel where $h_{i j}=d_{i j}^{-\gamma}$, with $d_{i j}$ being the distance between node $i$ and $j$ and $\gamma>1$ is the channel attenuation coefficient. In the second model, we assume that each channel experiences an independent phase fading, that is $h_{i j}=d_{i j}^{-\gamma} e^{j \theta_{i j}}$, where $\theta_{i j}$ is uniformly distributed over $[0,2 \pi)$. We believe that the second model is more practically relevant than the real channel scenario. 


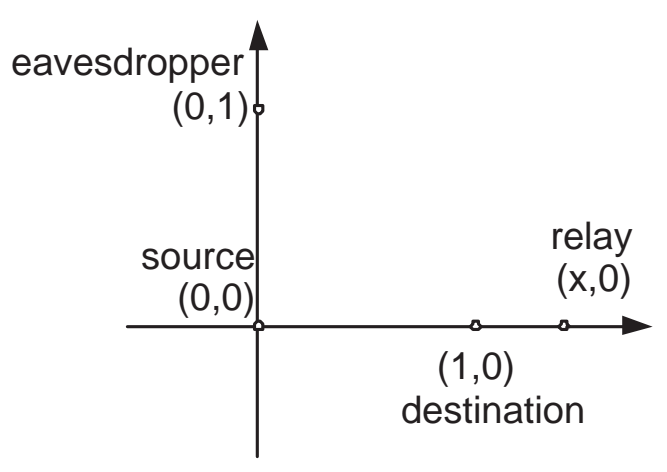

Fig. 4: The network topology.

Figure 3 shows the achievable perfect secrecy rate of the proposed schemes for the first channel model. In generating this figure, we use the network topology shown in Figure 4 , where we put the source at $(0,0)$, the destination at $(1,0)$, the eavesdropper at $(0,1)$, and the relay node at $(x, 0)$. We let $P_{1}=1, P_{2}=8$. Since $d_{s d}=d_{s w}$, the perfect secrecy capacity of the eavesdropper channel without the relay node is zero. But, as shown in the figure, we can achieve a positive secrecy rate by introducing a relay node. In computing the upper-bound, we set $V_{1} \sim \mathcal{N}\left(0, P_{1}\right), V_{2} \sim \mathcal{N}\left(0, P_{2}\right)$ with a correlation coefficient $\rho$, and maximize over $\rho \in[-1,1]$. Notice that the Gaussian input is not necessarily optimal for the upper-bound. We can see that, when the relay is near the source, the DF scheme touches the Gaussian upper-bound. Also, when $x>1$, it is clear that DF cooperation does not offer any gain, while NF and AF still offer positive rates. Notice that when $x>1$, both $d_{s r}, d_{s d}$ are larger than $d_{s w}$. The interesting observation here is that though both the destination and relay are in disadvantage positions compared with the eavesdropper, they can cooperate with each other and gain some advantage over the eavesdropper. If the relay is at 0 , our model is equivalent to the case where the source has two antennas. Notice that the upper-bound of the perfect secrecy capacity is zero under this scenario. Hence, increasing the number of transmitting antenna at the source does not increase the secrecy capacity under the real channel model. On the other hand, if there is a relay node at an appropriate position, we can exploit this relay node to establish a secure source-destination link.

In the second scenario, we assume that before transmission, the source knows the phases $\theta_{s r}, \theta_{s d}, \theta_{r d}$, but does not know $\theta_{s w}, \theta_{r w}$. The random phase will not affect the achievable perfect 


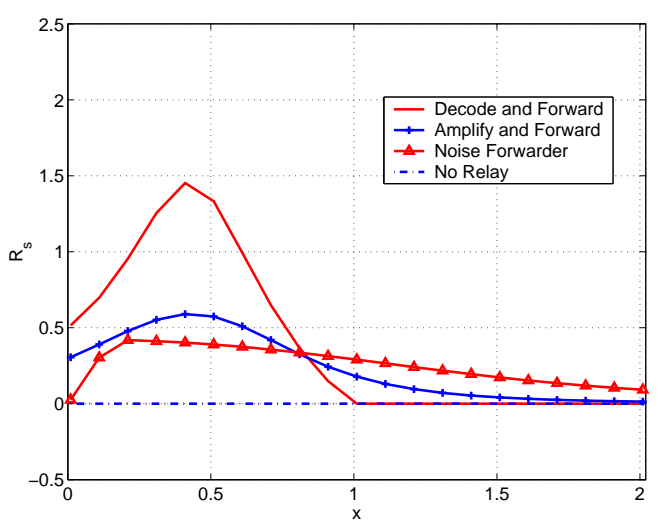

Fig. 5: The achievable perfect secrecy capacity for various schemes in the Gaussian relay eavesdropper channel with phase fading.

secrecy rate of NF since it does not depend on beam-forming between the source and relay. But, the rates of DF and AF are different here. In both cases, the source can adjust its phase according to the knowledge of the phase information about $\theta_{s r}, \theta_{s d}, \theta_{r d}$. In this way, the signals of the source and the relay will add up coherently at the destination, but not at the eavesdropper since $\theta_{s w}, \theta_{r w}$ are independent of $\theta_{s d}, \theta_{r d}, \theta_{s r}$. The secrecy rate of DF and AF could then be obtained by averaging (16), (20) over the random phases. Figure 5 shows the achievable perfect secrecy rates of the proposed strategies for the same setup as the first scenario. Due to the random phases, the achievable perfect secrecy capacity when the relay is at the same position as the source is not zero anymore. In this case, it will be beneficial to have multiple transmitting antennas at the source. Similar to the first scenario, when $x>1$, DF cooperation does not offer any benefit. But both NF and AF still enjoy non-zero secrecy rates.

\section{Conclusions}

In this paper, the relay-eavesdropper channel was studied. In particular, several cooperation strategies were proposed and the corresponding achievable performance bounds were obtained. Furthermore, an outer-bound on the optimal rate-equivocation region for this channel was developed. Of particular interest is the proposed NF strategy which was used to illustrate the deafhelper phenomenon, and to demonstrate the utility of the relay node in the reversely degraded relay-eavesdropper channel. Overall, our results establish the critical role of user cooperation in 
facilitating secure wireless communications and shed light on the unique feature of the relayeavesdropper channel.

Among the many open problems posed by our work, how to close the gap between the achievable performance and the outer-bound is arguably the most important one. This problem is expected to be challenging since the capacity of the classical relay channel remains unknown. The investigation of the role of feedback in the relay-eavesdropper channel is another interesting problem. In the relay channel without security constraints, noiseless/noisy feedback was shown to be beneficial. On the other hand, in the presence of an eavesdropper, the role and optimal mechanism of feedback is not yet known, since the eavesdropper could also benefit from the feedback signal. Finally, extending our work to a large scale network is expected to be of practical significance.

\section{APPENDIX I}

PROOF OF THEOREM 1

The proof follows that of [3].

$$
\begin{aligned}
n R_{e} & =H\left(W_{1} \mid Y_{2}^{n}\right) \\
& =H\left(W_{1}\right)-I\left(W_{1} ; Y_{2}^{n}\right) \\
& =I\left(W_{1} ; Y^{n}\right)-I\left(W_{1} ; Y_{2}^{n}\right)+H\left(W_{1} \mid Y^{n}\right) \\
& \leq \sum_{i=1}^{n}\left[I\left(W_{1} ; Y_{i} \mid Y^{i-1}\right)-I\left(W_{1} ; Y_{2, i} \mid Y_{2, i+1}^{n}\right)\right]+n \delta_{n}
\end{aligned}
$$

where $Y^{i-1}=Y(1, \cdots, i-1), Y_{2, i+1}^{n}=Y_{2}(i+1, \cdots, n)$, and $\delta_{n} \rightarrow 0$ as $n \rightarrow \infty$. We get this by using the chain rule to expand $I\left(W_{1} ; Y^{n}\right)$ from $i=1$ and expand $I\left(W_{1} ; Y_{2}^{n}\right)$ from $i=n$, also we use the Fano's inequality to bound $H\left(W_{1} \mid Y^{n}\right)$.

We continue

$$
\begin{aligned}
n R_{e} \leq & \sum_{i=1}^{n}\left[I\left(W_{1} ; Y_{i} \mid Y^{i-1}\right)-I\left(W_{1} ; Y_{2, i} \mid Y_{2, i+1}^{n}\right)\right]+n \delta_{n} \\
= & \sum_{i=1}^{n}\left[I\left(W_{1}, Y_{2, i+1}^{n} ; Y_{i} \mid Y^{i-1}\right)-I\left(Y_{2, i+1}^{n} ; Y_{i} \mid Y^{i-1}, W_{1}\right)\right. \\
& \left.-I\left(W_{1}, Y^{i-1} ; Y_{2, i} \mid Y_{2, i+1}^{n}\right)+I\left(Y^{i-1} ; Y_{2, i} \mid Y_{2, i+1}^{n}, W_{1}\right)\right]+n \delta_{n} \\
= & \sum_{i=1}^{n}\left[I\left(W_{1}, Y_{2, i+1}^{n} ; Y_{i} \mid Y^{i-1}\right)-I\left(W_{1}, Y^{i-1} ; Y_{2, i} \mid Y_{2, i+1}^{n}\right)\right]+n \delta_{n}
\end{aligned}
$$


since $\sum_{i=1}^{n} I\left(Y_{2, i+1}^{n} ; Y_{i} \mid Y^{i-1}, W_{1}\right)=\sum_{i=1}^{n} I\left(Y^{i-1} ; Y_{2, i} \mid Y_{2, i+1}^{n}, W_{1}\right)$, which is proved in the lemma 7 of [3]. Now

$$
\begin{aligned}
n R_{e} \leq & \sum_{i=1}^{n}\left[I\left(W_{1}, Y_{2, i+1}^{n} ; Y_{i} \mid Y^{i-1}\right)-I\left(W_{1}, Y^{i-1} ; Y_{2, i} \mid Y_{2, i+1}^{n}\right)\right]+n \delta_{n} \\
= & \sum_{i=1}^{n}\left[I\left(Y_{2, i+1}^{n} ; Y_{i} \mid Y^{i-1}\right)+I\left(W_{1} ; Y_{i} \mid Y^{i-1}, Y_{2, i+1}^{n}\right)\right. \\
& \left.-I\left(Y^{i-1} ; Y_{2, i} \mid Y_{2, i+1}^{n}\right)-I\left(W_{1} ; Y_{2, i} \mid Y^{i-1}, Y_{2, i+1}^{n}\right)\right]+n \delta_{n} \\
= & \sum_{i=1}^{n}\left[I\left(W_{1} ; Y_{i} \mid Y^{i-1}, Y_{2, i+1}^{n}\right)-I\left(W_{1} ; Y_{2, i} \mid Y^{i-1}, Y_{2, i+1}^{n}\right)\right]+n \delta_{n}
\end{aligned}
$$

since $\sum_{i=1}^{n} I\left(Y_{2, i+1}^{n} ; Y_{i} \mid Y^{i-1}\right)=\sum_{i=1}^{n} I\left(Y^{i-1} ; Y_{2, i} \mid Y_{2, i+1}^{n}\right)$, which is also proved in [3].

Now, let $J$ be a random variable uniformly distributed over $\{1, \cdots, n\}$, set $U=J Y^{i-1} Y_{2, i+1}^{n}, V_{1}=$ $J Y_{2, i+1}^{n} W_{1}, V_{2}=J Y^{i-1}, Y_{1}=Y_{1, J}, Y_{2}=Y_{2, J}, Y=Y_{J}, X_{1}=X_{1, J}, X_{2}=X_{2, J}$ we have

$$
\begin{aligned}
R_{e} & \leq \frac{1}{n} \sum_{i=1}^{n}\left[I\left(W_{1} ; Y_{i} \mid Y^{i-1}, Y_{2, i+1}^{n}\right)-I\left(W_{1} ; Y_{2, i} \mid Y^{i-1}, Y_{2, i+1}^{n}\right)\right]+\delta_{n} \\
& =\frac{1}{n} \sum_{i=1}^{n}\left[I\left(W_{1}, Y^{i-1}, Y_{2, i+1}^{n} ; Y_{i} \mid Y^{i-1}, Y_{2, i+1}^{n}\right)-I\left(W_{1}, Y^{i-1}, Y_{2, i+1}^{n} ; Y_{2, i} \mid Y^{i-1}, Y_{2, i+1}^{n}\right)\right]+\delta_{n} \\
& =I\left(V_{1}, V_{2} ; Y \mid U\right)-I\left(V_{1}, V_{2} ; Y_{2} \mid U\right)+\delta_{n} .
\end{aligned}
$$

Since the channel is memoryless, one can then check that $U \rightarrow\left(V_{1}, V_{2}\right) \rightarrow\left(X_{1}, X_{2}\right) \rightarrow$ $\left(Y, Y_{1}, Y_{2}\right)$ is a Markov chain. In the following, we bound $R_{1}$.

$$
\begin{aligned}
I\left(W_{1} ; \mathbf{Y}\right) & =\sum_{i=1}^{n} I\left(W_{1} ; Y_{i} \mid Y^{i-1}\right) \\
& =\sum_{i=1}^{n}\left[H\left(Y_{i} \mid Y^{i-1}\right)-H\left(Y_{i} \mid W_{1}, Y^{i-1}\right)\right] \\
& \leq \sum_{i=1}^{n}\left[H\left(Y_{i}\right)-H\left(Y_{i} \mid W_{1}, Y^{i-1}\right)\right] \\
& \leq \sum_{i=1}^{n}\left[H\left(Y_{i}\right)-H\left(Y_{i} \mid W_{1}, Y^{i-1}, Y_{2, i+1}^{n}\right)\right] \\
& =\sum_{i=1}^{n} I\left(W_{1}, Y^{i-1}, Y_{2, i+1}^{n} ; Y_{i}\right) .
\end{aligned}
$$

Hence

$$
R_{1} \leq \frac{1}{n} I\left(W_{1} ; \mathbf{Y}\right) \leq I\left(V_{1}, V_{2} ; Y\right)
$$


Also

$$
\begin{aligned}
I\left(W_{1} ; \mathbf{Y}\right) & =\sum_{i=1}^{n} I\left(W_{1} ; Y_{i} \mid Y^{i-1}\right) \\
& \leq \sum_{i=1}^{n} I\left(W_{1} ; Y_{i}, Y_{1, i} \mid Y^{i-1}\right) \\
& =\sum_{i=1}^{n}\left[H\left(Y_{i}, Y_{1, i} \mid Y^{i-1}\right)-H\left(Y_{i}, Y_{1, i} \mid W_{1}, Y^{i-1}\right)\right] \\
& \leq \sum_{i=1}^{n}\left[H\left(Y_{i}, Y_{1, i} \mid Y^{i-1}\right)-H\left(Y_{i}, Y_{1, i} \mid W_{1}, Y^{i-1}, Y_{2, i+1}^{n}\right)\right] \\
& =\sum_{i=1}^{n} I\left(W_{1}, Y_{2, i+1}^{n} ; Y_{i}, Y_{1, i} \mid Y^{i-1}\right) .
\end{aligned}
$$

Hence, we have

$$
R_{1} \leq \frac{1}{n} I\left(W_{1} ; \mathbf{Y}\right)=I\left(V_{1} ; Y, Y_{1} \mid V_{2}\right)
$$

So, we have

$$
R_{1} \leq \min \left\{I\left(V_{1}, V_{2} ; Y\right), I\left(V_{1} ; Y, Y_{1} \mid V_{2}\right)\right\}
$$

The claim is proved.

\section{APPENDIX II \\ Proof of THEOREM 2}

The proof is a combination of the coding schemes of Csiszár et. al. [3] and the regular coding and backward decoding scheme in the relay channel [7], [20]. We first replace $V_{1}, V_{2}$ in Theorem 2 with $X_{1}, X_{2}$. After proving Theorem 2 with $V_{1}, V_{2}$ replaced by $X_{1}, X_{2}$, we then prefix a memoryless channel with input $V_{1}, V_{2}$ and transmission probability $p\left(x_{1}, x_{2} \mid v_{1}, v_{2}\right)$ as reasoned in [3] to finish our proof.

\section{1) Codebook generation:}

For a given distribution $p\left(x_{1}, x_{2}\right)$, we first generate at random $2^{n R}$ i.i.d $n$-sequence at the relay node each drawn according to $p\left(\mathbf{x}_{2}\right)=\prod_{i=1}^{n} p\left(x_{2, i}\right)$, index them as $\mathbf{x}_{2}(a), a \in$ $\left[1,2^{n R}\right]$, where $R=\min \left\{I\left(X_{1}, X_{2} ; Y\right), I\left(X_{1} ; Y_{1} \mid X_{2}\right)\right\}-\epsilon_{0}$. For each $\mathbf{x}_{2}(a)$, generate $2^{n R}$ conditionally independent $n$-sequence $\mathbf{x}_{1}(k, a), k \in\left[1,2^{n R}\right]$ drawn randomly according to $p\left(\mathbf{x}_{1} \mid \mathbf{x}_{2}(a)\right)=\prod_{i=1}^{n} p\left(x_{1, i} \mid x_{2, i}(a)\right)$. Define $\mathcal{W}=\left\{1, \cdots, 2^{n\left[R-I\left(X_{1}, X_{2} ; Y_{2}\right)\right]}\right\}, \mathcal{L}=$ $\left\{1, \cdots, 2^{n I\left(X_{1}, X_{2} ; Y_{2}\right)}\right\}$ and $\mathcal{K}=\mathcal{W} \times \mathcal{L}=\left\{1, \cdots, 2^{n R}\right\}$. 


\section{2) Encoding}

We exploit the block Markov coding scheme, as argued in [4], the loss induced by this scheme is negligible as the number of blocks $B \rightarrow \infty$.

For a given rate pair $\left(R_{1}, R_{e}\right)$ with $R_{1} \leq R$ and $R_{e} \leq R_{1}$, we give the following coding strategy. Let the message to be transmitted at block $i$ be $w_{1}(i) \in \mathcal{W}_{1}=\{1, \cdots, M\}$, where $M=2^{n R_{1}}$.

The stochastic encoder at the transmitter first forms the following mappings.

- If $R_{1}>R-I\left(X_{1}, X_{2} ; Y_{2}\right)$, then we let $\mathcal{W}_{1}=\mathcal{W} \times \mathcal{J}$, where $\mathcal{J}=\left\{1, \cdots, 2^{n\left(R_{1}-\left[R-I\left(X_{1}, X_{2} ; Y_{2}\right)\right]\right)}\right\}$.

We let $g_{1}$ be the partition that partitions $\mathcal{L}$ into $|\mathcal{J}|$ equal size subsets. The stochastic encoder at transmitter will choose a mapping for each message $w_{1}(i)=(w(i), j(i)) \rightarrow$ $(w(i), l(i))$, where $l(i)$ is chosen randomly from the set $g_{1}^{-1}(j(i)) \subset \mathcal{L}$ with uniform distribution.

- If $R_{1}<R-I\left(X_{1}, X_{2} ; Y_{2}\right)$, the stochastic encoder will choose a mapping $w_{1}(i) \rightarrow$ $\left(w_{1}(i), l(i)\right)$, where $l(i)$ is chosen uniformly from the set $\mathcal{L}$.

Assume that the message $w_{1}(i-1)$ transmitted at block $i-1$ is associated with $(w(i-$ $1), l(i-1))$ and the message $w_{1}(i)$ intended to send at block $i$ is associated with $(w(i), l(i))$ by the stochastic encoder at the transmitter. We let $a(i-1)=(w(i-1), l(i-1))$ and $b(i)=(w(i), l(i))$. The encoder then sends $\mathbf{x}_{1}(b(i), a(i-1))$. The relay has an estimation $\hat{\hat{a}}(i-1)$ (see the decoding part), and thus sends the corresponding codeword $\mathbf{x}_{2}(\hat{\hat{a}}(i-1))$. At block 1 , the source sends $\mathbf{x}_{1}(b(1), 1)$, the relay sends $\mathbf{x}_{2}(1)$.

At block $B$, the source sends $\mathbf{x}_{1}(1, a(B-1))$, and the relay sends $\mathbf{x}_{2}(\hat{\hat{a}}(B-1))$.

\section{3) Decoding}

At the end of block $i$, the relay already has an estimation of the $\hat{\hat{a}}(i-1)$, which was sent at block $i-1$, and will declare that it receives $\hat{\hat{a}}(i)$, if this is the only pair such that $\left(\mathbf{x}_{1}(\hat{\hat{a}}(i), \hat{\hat{a}}(i-1)), \mathbf{x}_{2}(\hat{\hat{a}}(i-1)), \mathbf{y}_{1}(i)\right)$ are jointly typical. Since $R=\min \left\{I\left(X_{1} ; Y_{1} \mid X_{2}\right)\right.$, $\left.I\left(X_{1}, X_{2} ; Y\right)\right\}-\epsilon \leq I\left(X_{1} ; Y_{1} \mid X_{2}\right)-\epsilon$, then based on the AEP, one has $\hat{\hat{a}}(i)=a(i)$ with probability goes to 1 .

The destination decodes from the last block, $i$.e. block $B$. Suppose that at the end of block $B-1$, the relay decodes successfully, then the destination will declare that $\hat{a}(B-1)$ is received, if $\left(\mathbf{x}_{1}(1, \hat{a}(B-1)), \mathbf{x}_{2}(\hat{a}(B-1)), \mathbf{y}\right)$ are jointly typical. It's easy to see that if 
$R \leq I\left(X_{1}, X_{2} ; Y\right)$, we will have $\hat{a}(B-1)=a(B-1)$ with probability goes to 1 , as $n$ increases.

After getting $\hat{a}(B-1)$, the receiver can get an estimation of $a(i), i \in[1, B-2]$ in a similar way.

Having $\hat{a}(i-1)$, the destination can get the estimation of the message $w_{1}(i-1)$ by letting 1) $\hat{w}_{1}(i-1)=(\hat{w}(i-1), \hat{j}(i-1))=\left(\hat{w}(i-1), g_{1}(\hat{l}(i-1))\right)$ if $R_{1}>R-I\left(X_{1}, X_{2} ; Y_{2}\right)$, 2) $\hat{w}_{1}(i-1)=\hat{w}(i-1)$ if $R_{1}<R-I\left(X_{1}, X_{2} ; Y_{2}\right)$.

The probability that $\hat{w}_{1}(i-1)=w_{1}(i-1)$ goes to one for sufficiently large $n$.

\section{4) Equivocation Computation}

$$
\begin{aligned}
H\left(W_{1} \mid \mathbf{Y}_{2}\right) & =H\left(W_{1}, \mathbf{Y}_{2}\right)-H\left(\mathbf{Y}_{2}\right) \\
& =H\left(W_{1}, \mathbf{Y}_{2}, \mathbf{X}_{1}, \mathbf{X}_{2}\right)-H\left(\mathbf{X}_{1}, \mathbf{X}_{2} \mid W_{1}, \mathbf{Y}_{2}\right)-H\left(\mathbf{Y}_{2}\right) \\
& =H\left(\mathbf{X}_{1}, \mathbf{X}_{2}\right)+H\left(W_{1}, \mathbf{Y}_{2} \mid \mathbf{X}_{1}, \mathbf{X}_{2}\right)-H\left(\mathbf{X}_{1}, \mathbf{X}_{2} \mid W_{1}, \mathbf{Y}_{2}\right)-H\left(\mathbf{Y}_{2}\right) \\
& \geq H\left(\mathbf{X}_{1}\right)+H\left(\mathbf{Y}_{2} \mid \mathbf{X}_{1}, \mathbf{X}_{2}\right)-H\left(\mathbf{X}_{1}, \mathbf{X}_{2} \mid W_{1}, \mathbf{Y}_{2}\right)-H\left(\mathbf{Y}_{2}\right)
\end{aligned}
$$

First, let us calculate $H\left(\mathbf{X}_{1}, \mathbf{X}_{2} \mid W_{1}, \mathbf{Y}_{2}\right)$. Given $W_{1}$, the eavesdropper can also do backward decoding as the receiver. At the end of block $B$, given $W_{1}$, the eavesdropper knows $w(B-1)$, hence it will decode $l(B-1)$, by letting $l(B-1)=\hat{l}(B-1)$, if $\hat{l}(B-1)$ is the only one such that $\left(\mathbf{x}_{1}(1,(w(B-1), \hat{l}(B-1))), \mathbf{x}_{2}((w(B-1), \hat{l}(B-1))), \mathbf{y}\right)$ are jointly typical. Since $l \in\left[1,2^{n I\left(X_{1}, X_{2} ; Y_{2}\right)}\right]$, we have

$$
\begin{aligned}
& \operatorname{Pr}\left\{\left(\mathbf{X}_{1}(1, a(i-1)), \mathbf{X}_{2}(\hat{\hat{a}}(i-1))\right)\right. \\
& \left.\quad \neq\left(\mathbf{X}_{1}(1,(w(B-1), \hat{l}(B-1))), \mathbf{X}_{2}((w(B-1), \hat{l}(B-1)))\right)\right\} \leq \epsilon_{1} .
\end{aligned}
$$

Then based on Fano's inequality, we have

$$
\frac{1}{n} H\left(\mathbf{X}_{1}, \mathbf{X}_{2} \mid W_{1}=w_{1}, \mathbf{Y}_{2}\right) \leq \frac{1}{n}+\epsilon_{1} I\left(X_{1}, X_{2} ; Y_{2}\right)
$$

Hence, we have

$$
\frac{1}{n} H\left(\mathbf{X}_{1}, \mathbf{X}_{2} \mid W_{1}, \mathbf{Y}_{2}\right)=\frac{1}{n} \sum_{w_{1} \in \mathcal{W}_{1}} p\left(W_{1}=w_{1}\right) H\left(\mathbf{X}_{1}, \mathbf{X}_{2} \mid W_{1}=w_{1}, \mathbf{Y}_{2}\right) \leq \epsilon_{2}
$$

when $n$ is sufficiently large.

Since the channel is memoryless, we have $H\left(\mathbf{Y}_{2}\right)-H\left(\mathbf{Y}_{2} \mid \mathbf{X}_{1}, \mathbf{X}_{2}\right) \leq n I\left(X_{1}, X_{2} ; Y_{2}\right)+n \delta_{n}$, where $\delta_{n} \rightarrow 0$, as $n \rightarrow \infty[2]$. 
Now, from the code construction, we have $H\left(\mathbf{X}_{1}\right)=n R$ if $R_{1}>R-I\left(X_{1}, X_{2} ; Y_{2}\right)$. In this case, we get $n R_{e}=H\left(W_{1} \mid \mathbf{Y}_{2}\right) \geq n\left(R-I\left(X_{1}, X_{2} ; Y_{2}\right)-\epsilon_{3}\right)$. If $R_{1} \leq R-I\left(X_{1}, X_{2} ; Y_{2}\right)$, $H\left(\mathbf{X}_{1}\right)=n\left(R_{1}+I\left(X_{1}, X_{2} ; Y_{2}\right)\right)$, in this case, we get the perfect secrecy, since

$$
n R_{e} \geq n\left(R_{1}+I\left(X_{1}, X_{2} ; Y_{2}\right)\right)-n I\left(X_{1}, X_{2} ; Y_{2}\right)-n \epsilon_{3} \geq n\left(R_{1}-\epsilon_{3}\right)
$$

The claim is proved.

\section{APPENDIX III}

\section{PROOF OF THEOREM 3}

As [3], we first prove the result for the case where $V_{1}, V_{2}$ in Theorem 3 are replaced with $X_{1}, X_{2}$, then prefix a memoryless channel with transition probability $p\left(x_{1} \mid v_{1}\right) p\left(x_{2} \mid v_{2}\right)$ to finish our proof.

We first consider the case $I\left(X_{1} ; Y \mid X_{2}\right)<I\left(X_{1} ; Y_{2} \mid X_{2}\right)$, i.e., the channel between the source and the eavesdropper is better than the channel between the source and the destination. In this case, we only need to consider $\min \left\{I\left(X_{2} ; Y\right), I\left(X_{2} ; Y_{2}\right)\right\}=I\left(X_{2} ; Y_{2}\right)$, otherwise, the secrecy rate will be zero. Thus in this case, the last equation in (9) changes to $R_{e}<\left[I\left(X_{1} ; Y \mid X_{2}\right)+\right.$ $\left.\min \left\{I\left(X_{2} ; Y\right), I\left(X_{2} ; Y_{2} \mid X_{1}\right)\right\}-I\left(X_{1}, X_{2} ; Y_{2}\right)\right]^{+}$.

\section{1) Codebook generation:}

For a given distribution $p\left(x_{1}\right) p\left(x_{2}\right)$, we generate at random $2^{n R_{2}}$ i.i.d $n$-sequence at the relay node each drawn according to $p\left(\mathbf{x}_{2}\right)=\prod_{i=1}^{n} p\left(x_{2, i}\right)$, index them as $\mathbf{x}_{2}(a), a \in$ $\left[1,2^{n R_{2}}\right]$, where we set $R_{2}=\min \left\{I\left(X_{2} ; Y\right), I\left(X_{2} ; Y_{2} \mid X_{1}\right)\right\}-\epsilon$. We also generate random $2^{n R}$ i.i.d $n$-sequence at the source each drawn according to $p\left(\mathbf{x}_{1}\right)=\prod_{i=1}^{n} p\left(x_{1, i}\right)$, index them as $\mathbf{x}_{1}(k), k \in\left[1,2^{n R}\right]$, where $R=I\left(X_{1} ; Y \mid X_{2}\right)-\epsilon$. Let

$$
R^{\prime}=\min \left\{I\left(X_{2} ; Y\right), I\left(X_{2} ; Y_{2} \mid X_{1}\right)\right\}+I\left(X_{1} ; Y \mid X_{2}\right)-I\left(X_{1}, X_{2} ; Y_{2}\right)
$$

and define $\mathcal{W}=\left\{1, \cdots, 2^{n R^{\prime}}\right\}, \mathcal{L}=\left\{1, \cdots, 2^{n\left(R-R^{\prime}\right)}\right\}$ and $\mathcal{K}=\mathcal{W} \times \mathcal{L}=\left\{1, \cdots, 2^{n R}\right\}$.

\section{2) Encoding}

For a given rate pair $\left(R_{1}, R_{e}\right)$ with $R_{1} \leq R, R_{e} \leq R_{1}$, we give the following coding strategy. Let the message to be transmitted at block $i$ be $w_{1}(i) \in \mathcal{W}_{1}=[1, M]$, where $M=2^{n R_{1}}$.

The stochastic encoder at the transmitter first forms the following mappings. 
- If $R_{1}>R^{\prime}$, then we let $\mathcal{W}_{1}=\mathcal{W} \times \mathcal{J}$, where $\mathcal{J}=\left\{1,2^{n\left(R_{1}-R^{\prime}\right)}\right\}$. We let $g_{1}$ be the partition that partitions $\mathcal{L}$ into $|\mathcal{J}|$ equal size subsets. The stochastic encoder at transmitter will choose a mapping for each message $w_{1}(i)=(w(i), j(i)) \rightarrow(w(i), l(i))$, where $l(i)$ is chosen randomly from the set $g_{1}^{-1}(j(i)) \subset \mathcal{L}$ with uniform distribution.

- If $R_{1}<R^{\prime}$, the stochastic encoder will choose a mapping $w_{1}(i) \rightarrow\left(w_{1}(i), l(i)\right)$, where $l(i)$ is chosen uniformly from the set $\mathcal{L}$.

Suppose the message $w_{1}(i)$ intended to send at block $i$ is associated with $(w(i), l(i))$ by the stochastic encoder at the transmitter. The encoder then sends $\mathbf{x}_{1}((w(i), l(i)))$. The relay uniformly picks a code $\mathbf{x}_{2}(a)$ from $a \in\left[1, \cdots, 2^{n R_{2}}\right]$, and sends $\mathbf{x}_{2}(a)$.

\section{3) Decoding}

At the end of block $i$, the destination declares that $\hat{a}(i)$ is received, if $\hat{a}(i)$ is the only one such that $\left(\mathbf{x}_{2}(\hat{a}(i)), \mathbf{y}\right)$ are jointly typical. If there does not exist or there exist more than one such sequences, the destination declares an error. Since $R_{2}=\min \left\{I\left(X_{2} ; Y\right), I\left(X_{2} ; Y_{2} \mid X_{1}\right)\right\}-$ $\epsilon \leq I\left(X_{2} ; Y\right)-\epsilon$, then based on AEP, we know that the error probability will be less than any given positive number $\epsilon$, when the codeword length $n$ is long enough.

The destination then declares that $\hat{k}$ is received, if $\hat{k}$ is the only one such that $\left(\mathbf{x}_{1}(\hat{k}), \mathbf{x}_{2}(\hat{a}), \mathbf{y}\right)$ are jointly typical, otherwise declares an error. Since $R=I\left(X_{1} ; Y \mid X_{2}\right)-\epsilon$, then based on AEP, we know that we will have error probability goes to zero, when $n$ is sufficiently large.

Having $\hat{k}(i)$, the destination can get the estimation of the message $w_{1}(i)$ by letting

1) $\hat{w}_{1}(i)=(\hat{w}(i), \hat{j}(i))=\left(\hat{w}(i), g_{1}(\hat{l}(i))\right)$, if $R_{1}>R^{\prime}$,

2) $\hat{w}_{1}(i)=\hat{w}(i)$, if $R_{1}<R^{\prime}$.

The probability that $\hat{w}_{1}(i)=w_{1}(i)$ goes to one for sufficiently large $n$.

\section{4) Equivocation Computation}

$$
\begin{aligned}
H\left(W_{1} \mid \mathbf{Y}_{2}\right) & =H\left(W_{1}, \mathbf{Y}_{2}\right)-H\left(\mathbf{Y}_{2}\right) \\
& =H\left(W_{1}, \mathbf{Y}_{2}, \mathbf{X}_{1}, \mathbf{X}_{2}\right)-H\left(\mathbf{X}_{1}, \mathbf{X}_{2} \mid W_{1}, \mathbf{Y}_{2}\right)-H\left(\mathbf{Y}_{2}\right) \\
& =H\left(\mathbf{X}_{1}, \mathbf{X}_{2}\right)+H\left(W_{1}, \mathbf{Y}_{2} \mid \mathbf{X}_{1}, \mathbf{X}_{2}\right)-H\left(\mathbf{X}_{1}, \mathbf{X}_{2} \mid W_{1}, \mathbf{Y}_{2}\right)-H\left(\mathbf{Y}_{2}\right) \\
& \geq H\left(\mathbf{X}_{1}, \mathbf{X}_{2}\right)+H\left(\mathbf{Y}_{2} \mid \mathbf{X}_{1}, \mathbf{X}_{2}\right)-H\left(\mathbf{X}_{1}, \mathbf{X}_{2} \mid W_{1}, \mathbf{Y}_{2}\right)-H\left(\mathbf{Y}_{2}\right)
\end{aligned}
$$

Now let's calculate $H\left(\mathbf{X}_{1}, \mathbf{X}_{2} \mid W_{1}, \mathbf{Y}_{2}\right)$. Given $W_{1}$, the eavesdropper can do joint decoding. 
At any block $i$, given $W_{1}$, the eavesdropper knows $w(i)$, hence it will decode $l(i)$ and $a(i)$ sent by the relay, by letting $l(i)=\hat{l}(i), a(i)=\hat{a}(i)$, if $\hat{l}(i), \hat{a}(i)$ are the only one such that $\left(\mathbf{x}_{1}(w(i), \hat{l}(i)), \mathbf{x}_{2}(\hat{a}(i)), \mathbf{y}\right)$ are jointly typical. Then, since $R_{2}=\min \left\{I\left(X_{2} ; Y\right), I\left(X_{2} ; Y_{2} \mid X_{1}\right)\right\}-$ $\epsilon \leq I\left(X_{2} ; Y_{2} \mid X_{1}\right)-\epsilon$, we get

$$
\begin{aligned}
\frac{1}{2} \log _{2}(|\mathcal{L}|)+R_{2}= & R+I\left(X_{1}, X_{2} ; Y_{2}\right)-\min \left\{I\left(X_{2} ; Y\right), I\left(X_{2} ; Y_{2} \mid X_{1}\right)\right\} \\
& -I\left(X_{1} ; Y \mid X_{2}\right)+\min \left\{I\left(X_{2} ; Y\right), I\left(X_{2} ; Y_{2} \mid X_{1}\right)\right\}-\epsilon \\
\leq & I\left(X_{1}, X_{2} ; Y_{2}\right)-\epsilon,
\end{aligned}
$$

Also, we have $\frac{1}{2} \log _{2}(|\mathcal{L}|)<R \leq I\left(X_{1} ; Y_{2} \mid X_{2}\right)-\epsilon$.

So $\operatorname{Pr}\left\{\left(\mathbf{X}_{1}(w(i), \hat{l}(i)), \mathbf{X}_{2}(\hat{a}(i))\right) \neq\left(\mathbf{X}_{1}(w(i), l(i)), \mathbf{X}_{2}(a(i))\right)\right\} \leq \epsilon_{1}$.

Then based on Fano's inequality, we have

$$
\frac{1}{n} H\left(\mathbf{X}_{1}, \mathbf{X}_{2} \mid W_{1}=w_{1}, \mathbf{Y}_{2}\right) \leq \frac{1}{n}+\epsilon_{1} I\left(X_{1}, X_{2} ; Y_{2}\right)
$$

Hence, we have

$$
\frac{1}{n} H\left(\mathbf{X}_{1}, \mathbf{X}_{2} \mid W_{1}, \mathbf{Y}_{2}\right)=\frac{1}{n} \sum_{w_{1} \in \mathcal{W}_{1}} p\left(W_{1}=w_{1}\right) H\left(\mathbf{X}_{1}, \mathbf{X}_{2} \mid W_{1}=w_{1}, \mathbf{Y}_{2}\right) \leq \epsilon_{2}
$$

when $n$ is sufficiently large.

Now, $H\left(\mathbf{Y}_{2}\right)-H\left(\mathbf{Y}_{2} \mid \mathbf{X}_{1}, \mathbf{X}_{2}\right) \leq n I\left(X_{1}, X_{2} ; Y_{2}\right)+n \delta_{n}$, where $\delta_{n} \rightarrow 0$, as $n \rightarrow \infty$.

Also we have $H\left(\mathbf{X}_{1}, \mathbf{X}_{2}\right)=H\left(\mathbf{X}_{1}\right)+H\left(\mathbf{X}_{2}\right)$ since $\mathbf{x}_{1}$ and $\mathbf{x}_{2}$ are independent. If $R_{1}>R^{\prime}$, we have $H\left(\mathbf{X}_{1}, \mathbf{X}_{2}\right)=R+R_{2}=I\left(X_{1} ; Y \mid X_{2}\right)+\min \left\{I\left(X_{2} ; Y\right), I\left(X_{2} ; Y_{2} \mid X_{1}\right)\right\}$. Combining these, we get $n R_{e}=H\left(W_{1} \mid \mathbf{Y}_{2}\right) \geq n\left(\min \left\{I\left(X_{2} ; Y\right), I\left(X_{2} ; Y_{2} \mid X_{1}\right)\right\}+I\left(X_{1} ; Y \mid X_{2}\right)\right)-$ $n I\left(X_{1}, X_{2} ; Y_{2}\right)-n \epsilon_{4}$.

On the other hand, if $R_{1}<R^{\prime}$, we have $H\left(\mathbf{X}_{1}\right)=R_{1}+I\left(X_{1}, X_{2} ; Y_{2}\right)-\min \left\{I\left(X_{2} ; Y\right), I\left(X_{2} ; Y_{2} \mid X_{1}\right)\right\}$, hence we have $H\left(\mathbf{X}_{1}, \mathbf{X}_{2}\right)=R_{1}+I\left(X_{1}, X_{2} ; Y_{2}\right)-\epsilon$. We get perfect secrecy rate, since $n R_{e}=H\left(W_{1} \mid \mathbf{Y}_{2}\right) \geq n R_{1}-n \epsilon_{4}$.

This case is proved.

Now, consider the case $I\left(X_{1} ; Y \mid X_{2}\right)>I\left(X_{1} ; Y_{2} \mid X_{2}\right)$. If $\min \left\{I\left(X_{2} ; Y\right), I\left(X_{2} ; Y_{2}\right)\right\}=I\left(X_{2} ; Y\right)$, then we have $\min \left\{I\left(X_{2} ; Y\right), I\left(X_{2} ; Y_{2} \mid X_{1}\right)\right\}=I\left(X_{2} ; Y\right)$, because $I\left(X_{2} ; Y_{2} \mid X_{1}\right)>I\left(X_{2} ; Y_{2}\right)$ since $X_{1}, X_{2}$ are independent. Under this case, we only need to prove $R_{e} \leq I\left(X_{1} ; Y \mid X_{2}\right)-$ $I\left(X_{1} ; Y_{2} \mid X_{2}\right)$ are achievable, which can be achieved by letting the codeword rate be $I\left(X_{1} ; Y \mid X_{2}\right)$ 
and $R^{\prime}=I\left(X_{1} ; Y \mid X_{2}\right)-I\left(X_{1} ; Y \mid X_{2}\right)$. Now the equivocation rate of the eavesdropper can be calculated as

$$
\begin{aligned}
H\left(W_{1} \mid \mathbf{Y}_{2}\right) & \geq H\left(W_{1} \mid \mathbf{Y}_{2}, \mathbf{X}_{2}\right) \\
& =H\left(W_{1}, \mathbf{Y}_{2} \mid \mathbf{X}_{2}\right)-H\left(\mathbf{Y}_{2} \mid \mathbf{X}_{2}\right) \\
& =H\left(W_{1}, \mathbf{Y}_{2}, \mathbf{X}_{1} \mid \mathbf{X}_{2}\right)-H\left(\mathbf{X}_{1} \mid W_{1}, \mathbf{Y}_{2}, \mathbf{X}_{2}\right)-H\left(\mathbf{Y}_{2} \mid \mathbf{X}_{2}\right) \\
& =H\left(\mathbf{X}_{1} \mid \mathbf{X}_{2}\right)+H\left(W_{1}, \mathbf{Y}_{2} \mid \mathbf{X}_{1}, \mathbf{X}_{2}\right)-H\left(\mathbf{X}_{1} \mid W_{1}, \mathbf{Y}_{2}, \mathbf{X}_{2}\right)-H\left(\mathbf{Y}_{2} \mid \mathbf{X}_{2}\right) \\
& \geq H\left(\mathbf{X}_{1}\right)+H\left(\mathbf{Y}_{2} \mid \mathbf{X}_{1}, \mathbf{X}_{2}\right)-H\left(\mathbf{X}_{1} \mid W_{1}, \mathbf{Y}_{2}, \mathbf{X}_{2}\right)-H\left(\mathbf{Y}_{2} \mid \mathbf{X}_{2}\right),
\end{aligned}
$$

since $\mathbf{x}_{1}, \mathbf{x}_{2}$ are independent. This can then be shown to be larger than $n\left(I\left(X_{1} ; Y \mid X_{2}\right)-\right.$ $\left.I\left(X_{1} ; Y_{2} \mid X_{2}\right)-\epsilon\right)$.

If $\min \left\{I\left(X_{2} ; Y\right), I\left(X_{2} ; Y_{2}\right)\right\}=I\left(X_{2} ; Y_{2}\right)$, the last line in (9) changes to $R_{e}<\left[I\left(X_{1} ; Y \mid X_{2}\right)+\right.$ $\left.\min \left\{I\left(X_{2} ; Y\right), I\left(X_{2} ; Y_{2} \mid X_{1}\right)\right\}-I\left(X_{1}, X_{2} ; Y_{2}\right)\right]^{+}$, then we can use a coding/decoding scheme similar to the one developed above to show the achievability.

The claim is achieved.

\section{APPENDIX IV}

\section{Proof of TheOrem 4}

The proof is a combination of the coding scheme of Csiszár et. al. [3] and a revised CF scheme in the relay channel [4].

\section{1) Codebook generation:}

We first generate at random $2^{n R}$ i.i.d $n$-sequence $\mathrm{x}_{1}$ at the source node each drawn according to $p\left(\mathbf{x}_{1}\right)=\prod_{j=1}^{n} p\left(x_{1, j}\right)$, index them as $\mathbf{x}_{1}(k), k \in\left[1,2^{n R}\right]$, with $R=I\left(X_{1} ; \hat{Y}_{1}, Y \mid X_{2}\right)-\epsilon$. Generate at random $2^{n R_{2}}$ i.i.d $n$-sequence $\mathbf{x}_{2}$ each with probability $p\left(\mathbf{x}_{2}\right)=\prod_{j=1}^{n} p\left(x_{2, j}\right)$. Index these as $\mathbf{x}_{2}(s), s \in\left[1,2^{n R_{2}}\right]$, where

$$
R_{2}=\min \left\{I\left(X_{2} ; Y\right), I\left(X_{2} ; Y_{2} \mid X_{1}\right)\right\}-\epsilon
$$

For each $\mathbf{x}_{2}(s)$, generate at random $2^{n\left(R_{2}-R_{0}\right)}$ i.i.d $\hat{\mathbf{y}}_{1}$, each with probability $p\left(\hat{\mathbf{y}}_{1} \mid \mathbf{x}_{2}(s)\right)=$ $\prod_{j=1}^{n} p\left(\hat{y}_{1, j} \mid x_{2, j}(s)\right)$. Label these $\hat{\mathbf{y}}_{1}(z, s), z \in\left[1,2^{n \hat{R}}\right], s \in\left[1,2^{n R_{2}}\right]$, where we set $\hat{R}=$ $R_{2}-R_{0}$. Equally divide these $2^{n R_{2}} \mathbf{x}_{2}$ sequences into $2^{n \hat{R}}$ bins, hence there are $2^{n R_{0}} \mathbf{x}_{2}$ sequences at each bin. Let $f$ be this mapping, that is $z=f(s)$. 
Let $R^{\prime}=\min \left\{I\left(X_{2} ; Y\right), I\left(X_{2} ; Y_{2} \mid X_{1}\right)\right\}+I\left(X_{1} ; \hat{Y}_{1}, Y \mid X_{2}\right)-I\left(X_{1}, X_{2} ; Y_{2}\right)$.

Define $\mathcal{W}=\left\{1, \cdots, 2^{n R^{\prime}}\right\}, \mathcal{L}=\left\{1, \cdots, 2^{n\left(R-R^{\prime}\right)}\right\}$ and $\mathcal{K}=\mathcal{W} \times \mathcal{L}=\left\{1, \cdots, 2^{n R}\right\}$.

\section{2) Encoding}

We exploit the block Markov coding scheme.

For a given rate pair $\left(R_{1}, R_{e}\right)$, where $R_{1} \leq R, R_{e} \leq R_{1}$, we give the following coding strategy. Let the message to be transmitted at block $i$ be $w_{1}(i) \in \mathcal{W}_{1}=[1, M]$, where $M=2^{n R_{1}}$. We require $R_{1} \leq R$.

The stochastic encoder at the transmitter first forms the following mappings.

- If $R_{1}>R^{\prime}$, we let $\mathcal{W}_{1}=\mathcal{W} \times \mathcal{J}$, where $\mathcal{J}=\left\{1,2^{n\left(R_{1}-R^{\prime}\right)}\right\}$. We let $g_{1}$ be the partition that partitions $\mathcal{L}$ into $|\mathcal{J}|$ equal size subsets. The stochastic encoder at transmitter will choose a mapping for each message $w_{1}(i)=(w(i), j(i)) \rightarrow(w(i), l(i))$, where $l(i)$ is chosen randomly from the set $g_{1}^{-1}(j(i)) \subset \mathcal{L}$ with uniform distribution.

- If $R_{1}<R^{\prime}$, the stochastic encoder will choose a mapping $w_{1}(i) \rightarrow\left(w_{1}(i), l(i)\right)$, where $l(i)$ is chosen uniformly from the set $\mathcal{L}$.

At first consider block $i$, where $i \neq 1, B$, which means it's not the first or the last block. Assume that the message $w_{1}(i)$ intended to send at block $i$ is associated with $(w(i), l(i))$ by the stochastic encoder at the transmitter. We let $k(i)=(w(i), l(i))$. Then the encoder at the source sends $\mathbf{x}_{1}(k(i))$ at block $i$. At the end of block $i-1$, we assume that $\left(\mathbf{x}_{2}(s(i-\right.$ $\left.1)), \hat{\mathbf{y}}_{1}(z(i-1), s(i-1)), \mathbf{y}_{1}(i-1)\right)$ are jointly typical 4 , then we choose $s(i)$ uniformly from bin $z(i-1)$, and the relay sends $\mathbf{x}_{2}(s(i))$ at block $i$.

When $i=1$, the source sends $\mathbf{x}_{1}(k(1))$, the relay sends $\mathbf{x}_{2}(1)$. When $i=B$, the source sends $\mathbf{x}_{1}(1)$, the relay sends $\mathbf{x}_{2}(s(B))$.

\section{3) Decoding}

First consider the relay node. At the end of block $i$, the relay already has $s(i)$, it then decides $z(i)$ by choosing $z(i)$ such that $\left(\mathbf{x}_{2}(s(i)), \hat{\mathbf{y}}_{1}(z(i), s(i)), \mathbf{y}_{1}(i)\right)$ are jointly typical. There exists such $z(i)$, if

$$
\hat{R} \geq I\left(Y_{1} ; \hat{Y}_{1} \mid X_{2}\right)
$$

and $n$ is sufficiently large. Choose $s(i+1)$ uniformly from bin $z(i)$.

\footnotetext{
${ }^{4}$ See the decoding part, such $z(i-1)$ exists.

${ }^{5}$ At the end of block 1 , relay knows $s(i)=1$, this is the starting point.
} 
The destination does backward decoding. The decoding process starts at the last block $B$, the destination decodes $s(B)$ by choosing unique $\hat{s}(B)$ such that $\left(\mathbf{x}_{2}(\hat{s}(B)), \mathbf{y}(B)\right)$ are jointly typical. We will have $\hat{s}(B)=s(B)$, if

$$
R_{2} \leq I\left(X_{2} ; Y\right)
$$

and $n$ is sufficiently large.

Next, the destination moves to the block $B-1$. Now it already has $s(B)$, hence we also have $z(B-1)=f(s(B))$. It first declares that $\hat{s}(B-1)$ is received, if $\hat{s}(B-1)$ is the unique one such that $\left(\mathbf{x}_{2}(\hat{s}(B-1)), \mathbf{y}(B-1)\right)$ are jointly typical. If (49) is satisfied, $\hat{s}(B-1)=s(B-1)$ with high probability. After knowing $\hat{s}(B-1)$, the destination gets an estimation of $\hat{k}(B-1)$, by picking the unique $\hat{k}(B-1)$ such that $\left(\mathbf{x}_{1}(\hat{k}(B-\right.$ $\left.1)), \hat{\mathbf{y}}_{1}(z(B-1), \hat{s}(B-1)), \mathbf{y}(B-1), \mathbf{x}_{2}(\hat{s}(B-1))\right)$ are jointly typical. We will have $\hat{k}(B-1)=k(B-1)$ with high probability, if

$$
R \leq I\left(X_{1} ; \hat{Y}_{1}, Y \mid X_{2}\right)
$$

and $n$ is sufficiently large.

When the destination moves to block $i$, the destination has $s(i+1)$ and hence $z(i)=f(s(i+$ $1))$. It first declares that $\hat{s}(i)$ is received, by choosing unique $\hat{s}(i)$ such that $\left(\mathbf{x}_{2}(\hat{s}(i)), \mathbf{y}(i)\right)$ are jointly typical. If (49) is satisfied, $\hat{s}(i)=s(i)$ with high probability. After knowing $\hat{s}(i)$, the destination declares that $\hat{k}(i)$ is received, if $\hat{k}(i)$ is the unique one such that $\left(\mathbf{x}_{1}(\hat{k}(i)), \hat{\mathbf{y}}_{1}(z(i), \hat{s}(i)), \mathbf{y}(i), \mathbf{x}_{2}(\hat{s}(i))\right)$ are jointly typical. If (50) is satisfied, $\hat{k}(i)=k(i)$ with high probability when $n$ is sufficiently large.

Having $\hat{k}(i)$, the destination can get the estimation of the message $w_{1}(i)$ by letting 1$)$ $\hat{w}_{1}(i)=(\hat{w}(i), \hat{j}(i))=\left(\hat{w}(i), g_{1}(\hat{l}(i))\right)$, if $\left.R_{1}>R-R^{\prime}, 2\right) \hat{w}_{1}(i)=\hat{w}(i)$, if $R_{1}<R-R^{\prime}$. The probability that $\hat{w}_{1}(i)=w_{1}(i)$ goes to one for sufficiently large $n$.

\section{4) Equivocation Computation}

$$
\begin{aligned}
H\left(W_{1} \mid \mathbf{Y}_{2}\right) & =H\left(W_{1}, \mathbf{Y}_{2}\right)-H\left(\mathbf{Y}_{2}\right) \\
& =H\left(W_{1}, \mathbf{Y}_{2}, \mathbf{X}_{1}, \mathbf{X}_{2}\right)-H\left(\mathbf{X}_{1}, \mathbf{X}_{2} \mid W_{1}, \mathbf{Y}_{2}\right)-H\left(\mathbf{Y}_{2}\right) \\
& =H\left(\mathbf{X}_{1}, \mathbf{X}_{2}\right)+H\left(W_{1}, \mathbf{Y}_{2} \mid \mathbf{X}_{1}, \mathbf{X}_{2}\right)-H\left(\mathbf{X}_{1}, \mathbf{X}_{2} \mid W_{1}, \mathbf{Y}_{2}\right)-H\left(\mathbf{Y}_{2}\right) \\
& \geq H\left(\mathbf{X}_{1}, \mathbf{X}_{2}\right)+H\left(\mathbf{Y}_{2} \mid \mathbf{X}_{1}, \mathbf{X}_{2}\right)-H\left(\mathbf{X}_{1}, \mathbf{X}_{2} \mid W_{1}, \mathbf{Y}_{2}\right)-H\left(\mathbf{Y}_{2}\right)
\end{aligned}
$$


Following [2], we will have $H\left(\mathbf{Y}_{2}\right)-H\left(\mathbf{Y}_{2} \mid \mathbf{X}_{1}, \mathbf{X}_{2}\right) \leq n I\left(X_{1}, X_{2} ; Y_{2}\right)+n \delta_{n}$, where $\delta_{n} \rightarrow 0$ as $n \rightarrow \infty$.

Now let's calculate $H\left(\mathbf{X}_{1}, \mathbf{X}_{2} \mid W_{1}, \mathbf{Y}_{2}\right)$. Given $W_{1}$, the eavesdropper can do joint decoding. It does backward decoding. We pick up the story at block $i$, we suppose it already decodes $s(i+1)$ and hence $z(i)=f(s(i+1))$. Given $W_{1}$, the eavesdropper knows $w(i)$, hence it will decode $l(i)$ and $s(i)$ sent by the relay, by letting $l(i)=\hat{l}(i), s(i)=\hat{s}(i)$, if $\hat{l}(i), \hat{s}(i)$ are the only ones such that $\left(\mathbf{x}_{1}(w(i), \hat{l}(i)), \mathbf{x}_{2}(\hat{s}(i)), \hat{\mathbf{y}}_{1}(z(i), \hat{s}(i)), \mathbf{y}_{2}(i)\right)$ are jointly typical. Then, if $R_{2} \leq I\left(X_{2} ; Y_{2} \mid X_{1}\right)$ and (50) is satisfied, we have

$$
\begin{aligned}
\frac{1}{2} \log _{2}(|\mathcal{L}|)+R_{2}= & R-R^{\prime}+R_{2} \\
= & R-\min \left\{I\left(X_{2} ; Y\right), I\left(X_{2} ; Y_{2} \mid X_{1}\right)\right\}-I\left(X_{1} ; \hat{Y}_{1}, Y \mid X_{2}\right) \\
& +I\left(X_{1}, X_{2} ; Y_{2}\right)+\min \left\{I\left(X_{2} ; Y\right), I\left(X_{2} ; Y_{2} \mid X_{1}\right)\right\} \\
\leq & I\left(X_{1}, X_{2} ; Y_{2}\right) .
\end{aligned}
$$

Also, we have

$$
\frac{1}{2} \log _{2}(|\mathcal{L}|)<R \leq I\left(X_{1} ; \hat{Y}_{1}, Y_{2} \mid X_{2}\right)
$$

Thus, we have

$$
\operatorname{Pr}\left\{\left(\mathbf{X}_{1}(w(i), \hat{l}(i)), \mathbf{X}_{2}(\hat{s}(i))\right) \neq\left(\mathbf{X}_{1}(w(i), l(i)), \mathbf{X}_{2}(s(i))\right)\right\} \leq \epsilon_{1} .
$$

Then based on Fano's inequality, we have

$$
\frac{1}{n} H\left(\mathbf{X}_{1}, \mathbf{X}_{2}, \mid W_{1}=w_{1}, \mathbf{Y}_{2}\right) \leq \frac{1}{n}+\epsilon_{1} I\left(X_{1}, X_{2} ; Y_{2}\right)
$$

Hence, we have

$$
\frac{1}{n} H\left(\mathbf{X}_{1}, \mathbf{X}_{2} \mid W_{1}, \mathbf{Y}_{2}\right)=\frac{1}{n} \sum_{w_{1} \in \mathcal{W}_{1}} p\left(W_{1}=w_{1}\right) H\left(\mathbf{X}_{1}, \mathbf{X}_{2} \mid W_{1}=w_{1}, \mathbf{Y}_{2}\right) \leq \epsilon_{2}
$$

when $n$ is sufficiently large.

We know $H\left(\mathbf{X}_{1}, \mathbf{X}_{2}\right)=H\left(\mathbf{X}_{1}\right)+H\left(\mathbf{X}_{2} \mid \mathbf{X}_{1}\right) \geq n\left(R+R_{0}\right)$.

If $R_{1}>R^{\prime}$, we have $H\left(\mathbf{X}_{1}\right)=n R$, then we get

$$
n R_{e}=H\left(W_{1} \mid \mathbf{Y}_{2}\right) \geq n\left(R_{0}+I\left(X_{1} ; \hat{Y}_{1}, Y \mid X_{2}\right)-I\left(X_{1}, X_{2} ; Y_{2}\right)-\epsilon_{4}\right) .
$$

If $R_{1}<R^{\prime}$, we have $H\left(\mathbf{X}_{1}\right)=n\left(R_{1}+R-R^{\prime}\right)$, hence

$$
\left.n R_{e}=H\left(W_{1} \mid \mathbf{Y}_{2}\right) \geq n R_{1}+n\left(R_{0}-\min \left\{I\left(X_{2} ; Y\right), I\left(X_{2} ; Y_{2} \mid X_{1}\right)\right)\right\}-\epsilon_{4}\right) .
$$

The claim is proved. 


\section{APPENDIX V \\ PROOF OF THEOREM 5}

The proof follows closely with that of Theorem 3 , We first consider the case $I\left(X_{1} ; Y \mid X_{2}\right)<$ $I\left(X_{1} ; Y_{2} \mid X_{2}\right)$, i.e., the channel between the source and the eavesdropper is better than the channel between the source and the destination. In this case, we only need to consider the case $\min \left\{I\left(X_{2} ; Y\right), I\left(X_{2} ; Y_{2}\right)\right\}=I\left(X_{2} ; Y_{2}\right)$, otherwise, the perfect secrecy rate will be zero. Thus in this case, $R_{s 1}=\left[I\left(X_{1} ; Y \mid X_{2}\right)+\min \left\{I\left(X_{2} ; Y\right), I\left(X_{2} ; Y_{2} \mid X_{1}\right)\right\}-I\left(X_{1}, X_{2} ; Y_{2}\right)\right]^{+}$.

1) Codebook generation:

For a given distribution $p\left(x_{1}\right) p\left(x_{2}\right)$, we generate at random $2^{n R_{2}}$ i.i.d $n$-sequence at the relay node each drawn according to $p\left(\mathbf{x}_{2}\right)=\prod_{i=1}^{n} p\left(x_{2, i}\right)$, index them as $\mathbf{x}_{2}(a), a \in$ $\left[1,2^{n R_{2}}\right]$. Here we set $R_{2}=\min \left\{I\left(X_{2} ; Y\right), I\left(X_{2} ; Y_{2} \mid X_{1}\right)\right\}-\epsilon$. We also generate random $2^{n R}$ i.i.d $n$-sequence at the source each drawn according to $p\left(\mathbf{x}_{1}\right)=\prod_{i=1}^{n} p\left(x_{1, i}\right)$, index them as $\mathbf{x}_{1}(k), k \in\left[1,2^{n R}\right]$ with $R=I\left(X_{1} ; Y \mid X_{2}\right)-\epsilon$. Let

$$
R_{\text {min }}=\min \left\{R_{s 1}, R_{s 2}\right\}, R_{\max }=\max \left\{R_{s 1}, R_{s 2}\right\},
$$

where $R_{s 1}=\min \left\{I\left(X_{2} ; Y\right), I\left(X_{2} ; Y_{2} \mid X_{1}\right)\right\}+I\left(X_{1} ; Y \mid X_{2}\right)-I\left(X_{1}, X_{2} ; Y_{2}\right), R_{s 2}=I\left(X_{1} ; Y \mid X_{2}\right)-$ $I\left(X_{1} ; Y_{1} \mid X_{2}\right)$. We now define $\mathcal{W}=\left\{1, \cdots, 2^{n R_{\text {min }}}\right\}, \mathcal{L}_{1}=\left\{1, \cdots, 2^{n\left(R_{\text {max }}-R_{\text {min }}\right)}\right\}, \mathcal{L}_{2}=$ $\left\{1, \cdots, 2^{n\left(R-R_{\max }\right)}\right\}$ and $\mathcal{L}=\mathcal{L}_{1} \times \mathcal{L}_{2}, \mathcal{K}=\mathcal{W} \times \mathcal{L}$.

\section{2) Encoding}

Here, we consider perfect secrecy rate. For a given rate $R_{1} \leq R_{\text {min }}$, we give the following coding strategy to show that for any given $\epsilon \geq 0$, the equivocation rate at the eavesdropper and the relay node can be made to be larger or equal $R_{1}-\epsilon$.

Let the message to be transmitted at block $i$ be $w_{1}(i) \in \mathcal{W}_{1}=[1, M]$, where $M=$ $2^{n R_{1}}$. The stochastic encoder will choose a mapping $w_{1}(i) \rightarrow\left(w_{1}(i), l_{1}(i), l_{2}(i)\right)$, where $l_{1}(i), l_{2}(i)$ are chosen uniformly from the set $\mathcal{L}_{1}, \mathcal{L}_{2}$ respectively. We write $l(i)=\left(l_{1}(i), l_{2}(i)\right)$. Suppose the message $w_{1}(i)$ intended to send at block $i$ is associated with $(w(i), l(i))$ by the stochastic encoder at the transmitter. The encoder then sends $\mathbf{x}_{1}((w(i), l(i)))$. The relay uniformly picks a code $\mathbf{x}_{2}(a)$ from $a \in\left[1, \cdots, 2^{n R_{2}}\right]$, and sends $\mathbf{x}_{2}(a)$.

\section{3) Decoding}

At the end of block $i$, the destination declares that $\hat{a}(i)$ is received, if $\hat{a}(i)$ is the only one such that $\left(\mathbf{x}_{2}(\hat{a}(i)), \mathbf{y}\right)$ are jointly typical. If there does not exist or there exist more than one 
such sequences, the destination declares an error. Since $R_{2}=\min \left\{I\left(X_{2} ; Y\right), I\left(X_{2} ; Y_{2} \mid X_{1}\right)\right\}-$ $\epsilon \leq I\left(X_{2} ; Y\right)-\epsilon$, then based on AEP, we know that the error probability will be less than any given positive number $\epsilon$, when the codeword length $n$ is long enough.

The destination then declares that $\hat{k}$ is received, if $\hat{k}$ is the only one such that $\left(\mathbf{x}_{1}(\hat{k}), \mathbf{x}_{2}(\hat{a}), \mathbf{y}\right)$ are jointly typical, otherwise declares an error. Since $R=I\left(X_{1} ; Y \mid X_{2}\right)-\epsilon$, then based on AEP, we know that we will have error probability goes to zero, when $n$ is sufficiently large.

Having $\hat{k}(i)$, the destination can get the estimation of the message $w_{1}(i)$ by letting $\hat{w}_{1}(i)=$ $\hat{w}(i)$. The probability that $\hat{w}_{1}(i)=w_{1}(i)$ goes to one for sufficiently large $n$.

\section{4) Equivocation Computation}

We first calculate the equivocation rate of the eavesdropper when $R_{s 1} \leq R_{s 2}$.

$$
\begin{aligned}
H\left(W_{1} \mid \mathbf{Y}_{2}\right) & =H\left(W_{1}, \mathbf{Y}_{2}\right)-H\left(\mathbf{Y}_{2}\right) \\
& =H\left(W_{1}, \mathbf{Y}_{2}, \mathbf{X}_{1}, \mathbf{X}_{2}\right)-H\left(\mathbf{X}_{1}, \mathbf{X}_{2} \mid W_{1}, \mathbf{Y}_{2}\right)-H\left(\mathbf{Y}_{2}\right) \\
& =H\left(\mathbf{X}_{1}, \mathbf{X}_{2}\right)+H\left(W_{1}, \mathbf{Y}_{2} \mid \mathbf{X}_{1}, \mathbf{X}_{2}\right)-H\left(\mathbf{X}_{1}, \mathbf{X}_{2} \mid W_{1}, \mathbf{Y}_{2}\right)-H\left(\mathbf{Y}_{2}\right) \\
& \geq H\left(\mathbf{X}_{1}, \mathbf{X}_{2}\right)+H\left(\mathbf{Y}_{2} \mid \mathbf{X}_{1}, \mathbf{X}_{2}\right)-H\left(\mathbf{X}_{1}, \mathbf{X}_{2} \mid W_{1}, \mathbf{Y}_{2}\right)-H\left(\mathbf{Y}_{2}\right)
\end{aligned}
$$

Now let's calculate $H\left(\mathbf{X}_{1}, \mathbf{X}_{2} \mid W_{1}, \mathbf{Y}_{2}\right)$. Given $W_{1}$, the eavesdropper can do joint decoding. At any block $i$, given $W_{1}$, the eavesdropper knows $w(i)$, hence it will decode $l(i)=$ $\left(l_{1}(i), l_{2}(i)\right)$ and $a(i)$ sent by the relay, by letting $l(i)=\hat{l}(i), a(i)=\hat{a}(i)$, if $\hat{l}(i), \hat{a}(i)$ are the only one pair such that $\left(\mathbf{x}_{1}(w(i), \hat{l}(i)), \mathbf{x}_{2}(\hat{a}(i)), \mathbf{y}\right)$ are jointly typical. Since $R_{2}=$ $\min \left\{I\left(X_{2} ; Y\right), I\left(X_{2} ; Y_{2} \mid X_{1}\right)\right\}-\epsilon \leq I\left(X_{2} ; Y_{2} \mid X_{1}\right)-\epsilon$, we

$$
\begin{aligned}
\frac{1}{2} \log _{2}(|\mathcal{L}|)+R_{2}= & R+I\left(X_{1}, X_{2} ; Y_{2}\right)-\min \left\{I\left(X_{2} ; Y\right), I\left(X_{2} ; Y_{2} \mid X_{1}\right)\right\} \\
& -I\left(X_{1} ; Y \mid X_{2}\right)+\min \left\{I\left(X_{2} ; Y\right), I\left(X_{2} ; Y_{2} \mid X_{1}\right)\right\}-\epsilon \\
\leq & I\left(X_{1}, X_{2} ; Y_{2}\right)-\epsilon .
\end{aligned}
$$

Also, we have $\frac{1}{2} \log _{2}(|\mathcal{L}|)<R \leq I\left(X_{1} ; Y_{2} \mid X_{2}\right)$.

So $\operatorname{Pr}\left\{\left(\mathbf{X}_{1}(w(i), \hat{l}(i)), \mathbf{X}_{2}(\hat{a}(i))\right) \neq\left(\mathbf{X}_{1}(w(i), l(i)), \mathbf{X}_{2}(a(i))\right)\right\} \leq \epsilon_{1}$.

Then based on Fano's inequality, we have

$$
\frac{1}{n} H\left(\mathbf{X}_{1}, \mathbf{X}_{2} \mid W_{1}=w_{1}, \mathbf{Y}_{2}\right) \leq \frac{1}{n}+\epsilon_{1} I\left(X_{1}, X_{2} ; Y_{2}\right) .
$$


Hence, we have

$$
\frac{1}{n} H\left(\mathbf{X}_{1}, \mathbf{X}_{2} \mid W_{1}, \mathbf{Y}_{2}\right)=\frac{1}{n} \sum_{w_{1} \in \mathcal{W}_{1}} p\left(W_{1}=w_{1}\right) H\left(\mathbf{X}_{1}, \mathbf{X}_{2} \mid W_{1}=w_{1}, \mathbf{Y}_{2}\right) \leq \epsilon_{2}
$$

when $n$ is sufficiently large.

Now, $H\left(\mathbf{Y}_{2}\right)-H\left(\mathbf{Y}_{2} \mid \mathbf{X}_{1}, \mathbf{X}_{2}\right) \leq n I\left(X_{1}, X_{2} ; Y_{2}\right)+n \delta_{n}$, where $\delta_{n} \rightarrow 0$, as $n \rightarrow \infty$. Also we have $H\left(\mathbf{X}_{1}, \mathbf{X}_{2}\right)=H\left(\mathbf{X}_{1}\right)+H\left(\mathbf{X}_{2}\right)$ since $\mathbf{x}_{1}$ and $\mathbf{x}_{2}$ are independent. Now $H\left(\mathbf{X}_{1}\right)=R_{1}+I\left(X_{1}, X_{2} ; Y_{2}\right)-\min \left\{I\left(X_{2} ; Y\right), I\left(X_{2} ; Y_{2} \mid X_{1}\right)\right\}$, hence $H\left(\mathbf{X}_{1}, \mathbf{X}_{2}\right)=R_{1}+$ $I\left(X_{1}, X_{2} ; Y_{2}\right)-\epsilon$.

We get $n R_{e}=H\left(W_{1} \mid \mathbf{Y}_{2}\right) \geq n R_{1}-n \epsilon_{4}$.

Now we calculate the equivocation rate at the relay node.

$$
\begin{aligned}
H\left(W_{1} \mid \mathbf{Y}_{1}, \mathbf{X}_{2}\right) & \geq H\left(W_{1} \mid \mathbf{Y}_{1}, \mathbf{X}_{2}, L_{1}\right) \\
& =H\left(W_{1}, \mathbf{Y}_{1}, L_{1} \mid \mathbf{X}_{2}\right)-H\left(\mathbf{Y}_{1}, L_{1} \mid \mathbf{X}_{2}\right) \\
& =H\left(W_{1}, L_{1}, \mathbf{Y}_{1}, \mathbf{X}_{1} \mid \mathbf{X}_{2}\right)-H\left(\mathbf{X}_{1} \mid W_{1}, L_{1}, \mathbf{Y}_{1}, \mathbf{X}_{2}\right)-H\left(\mathbf{Y}_{1}, L_{1} \mid \mathbf{X}_{2}\right) \\
& =H\left(\mathbf{X}_{1} \mid \mathbf{X}_{2}\right)+H\left(W_{1}, L_{1}, \mathbf{Y}_{1} \mid \mathbf{X}_{1}, \mathbf{X}_{2}\right)-H\left(\mathbf{X}_{1} \mid W_{1}, L_{1}, \mathbf{Y}_{1}, \mathbf{X}_{2}\right)-H\left(\mathbf{Y}_{1}, L_{1} \mid \mathbf{X}_{2}\right) \\
& \stackrel{(a)}{\geq} H\left(\mathbf{X}_{1}\right)+H\left(\mathbf{Y}_{1} \mid \mathbf{X}_{1}, \mathbf{X}_{2}\right)-H\left(\mathbf{X}_{1} \mid W_{1}, L_{1}, \mathbf{Y}_{1}, \mathbf{X}_{2}\right)-H\left(L_{1}\right)-H\left(\mathbf{Y}_{1} \mid \mathbf{X}_{2}\right),
\end{aligned}
$$

where the first term of (a) comes from the fact that $\mathbf{x}_{1}, \mathbf{x}_{2}$ are independent, and the fourth term comes from the fact that $l_{1}, \mathbf{x}_{2}$ are independent.

Now, $H\left(L_{1}\right)=n\left(R_{\max }-R_{\text {min }}\right), H\left(\mathbf{Y}_{1} \mid \mathbf{X}_{1}, \mathbf{X}_{2}\right)-H\left(\mathbf{Y}_{1} \mid \mathbf{X}_{2}\right) \leq n I\left(X_{1} ; Y_{1} \mid X_{2}\right)+n \delta_{n}$. Given $w_{1}, l_{1}, \mathbf{x}_{2}$, the relay can just choose the $\mathbf{x}_{1}$ in the bin $\left(w_{1}, l_{1}\right)$ which is jointly typical with $\mathbf{x}_{2}, \mathbf{y}_{1}$. Since $\frac{1}{n} \log _{2}\left(\left|\mathcal{L}_{2}\right|\right) \leq I\left(X_{1} ; Y_{1} \mid X_{2}\right)$, we have $\operatorname{Pr}\left\{\hat{\mathbf{X}}_{1} \neq \mathbf{X}_{1}\right\} \leq \epsilon_{2}$.

Then based on Fano's inequality, we have

$$
\frac{1}{n} H\left(\mathbf{X}_{1} \mid W_{1}=w_{1}, L_{1}=l_{1}, \mathbf{Y}_{1}, \mathbf{X}_{2}=\mathbf{x}_{2}\right) \leq \frac{1}{n}+\epsilon_{1} I\left(X_{1} ; Y_{1} \mid X_{2}\right),
$$

Hence, we have

$$
\begin{aligned}
\frac{1}{n} H\left(\mathbf{X}_{1} \mid W_{1}, L_{1}, \mathbf{Y}_{1}, \mathbf{X}_{2}\right) & =\frac{1}{n} \sum_{w_{1}, l_{1}, \mathbf{x}_{2}} p\left(W_{1}=w_{1}, L_{1}=l_{1}, \mathbf{x}_{2}\right) H\left(\mathbf{X}_{1}, \mathbf{X}_{2} \mid W_{1}=w_{1}, L_{1}=l_{i}, \mathbf{x}_{2}, \mathbf{Y}_{1}\right) \\
& \leq \epsilon_{2}
\end{aligned}
$$

when $n$ is sufficiently large.

Also, based on the encoding part, we have $H\left(\mathbf{X}_{1}\right)=n\left(R_{1}+I\left(X_{1} ; Y \mid X_{2}\right)-R_{\text {min }}\right)$. 
Combining these, we get

$$
\begin{aligned}
H\left(W_{1} \mid \mathbf{Y}_{1}, \mathbf{X}_{2}\right) & \geq n\left(R_{1}+I\left(X_{1} ; Y \mid X_{2}\right)-R_{\text {min }}-\left(R_{\max }-R_{\min }\right)-I\left(X_{1} ; Y_{1} \mid X_{2}\right)-\delta_{n}\right) \\
& =n\left(R_{1}-\delta_{n}\right) .
\end{aligned}
$$

The equivocation rate of the relay and the eavesdropper when $R_{1 s} \geq R_{2 s}$ can be calculated similarly, with the only difference that we bound the equivocation rate of the eavesdropper by giving it $L_{1}$. This case is proved.

Now, consider the case $I\left(X_{1} ; Y \mid X_{2}\right)>I\left(X_{1} ; Y_{2} \mid X_{2}\right)$. If $\min \left\{I\left(X_{2} ; Y\right), I\left(X_{2} ; Y_{2}\right)\right\}=I\left(X_{2} ; Y\right)$, then we have $\min \left\{I\left(X_{2} ; Y\right), I\left(X_{2} ; Y_{2} \mid X_{1}\right)\right\}=I\left(X_{2} ; Y\right)$, because $I\left(X_{2} ; Y_{2} \mid X_{1}\right)>I\left(X_{2} ; Y_{2}\right)$ since $X_{1}, X_{2}$ are independent. Under this case, we only need to prove the case $R_{s 1}=\left[I\left(X_{1} ; Y \mid X_{2}\right)-\right.$ $\left.I\left(X_{1} ; Y_{2} \mid X_{2}\right)\right]^{+}$, which can be achieved by using a scheme similar to the one developed in proving (47). If $\min \left\{I\left(X_{2} ; Y\right), I\left(X_{2} ; Y_{2}\right)\right\}=I\left(X_{2} ; Y_{2}\right)$, and we only need to consider $R_{s 1}=$ $\left[I\left(X_{1} ; Y \mid X_{2}\right)+\min \left\{I\left(X_{2} ; Y\right), I\left(X_{2} ; Y_{2} \mid X_{1}\right)\right\}-I\left(X_{1}, X_{2} ; Y_{2}\right)\right]^{+}$, then we can use a coding/decoding scheme similar to the one developed above to show the achievability.

The claim is achieved.

\section{REFERENCES}

[1] C. E. Shannon, "Communication theory of secrecy systems," Bell System Technical Journal, vol. 28, pp. 656-715, Oct. 1949.

[2] A. D. Wyner, "The wire-tap channel," Bell System Technical Journal, vol. 54, no. 8, pp. 1355-1387, 1975.

[3] I. Csiszar and J. Korner, "Broadcast channels with confidential messages," IEEE Trans. on Information Theory, vol. 24, pp. 339-348, May 1978.

[4] T. Cover and A. E. Gamal, "Capacity theorems for the relay channel," IEEE Trans. on Information Theory, vol. 25, pp. 572-584, Sep. 1979.

[5] E. C. van der Meulen, “Three-terminal communication channels,” Adv. Appl. Probab., vol. 3, pp. 120-154, 1971.

[6] A. Sendonaris, E. Erkip, and B. Aazhang, "User cooperation diversity- part I: System description," IEEE Trans. on Communications, vol. 51, pp. 1927-1938, Nov. 2003.

[7] G. Kramer, M. Gastpar, and P. Gupta, "Cooperative strategies and capacity theorems for relay networks," IEEE Trans. on Information Theory, vol. 51, pp. 3037-3063, Sep. 2005.

[8] L. L. Xie and P. R. Kumar, "An achievable rate for the multiple-level relay channel," IEEE Trans. on Information Theory, vol. 51, pp. 1348-1358, Apr. 2005.

[9] L. Lai, K. Liu, and H. El-Gamal, “The three node wireless network: Achievable rates and cooperation strategies," IEEE Trans. on Information Theory, vol. 52, pp. 805-828, Mar. 2006.

[10] Y. Liang and V. V. Veeravalli, "Cooperative relay broadcast channels," IEEE Trans. on Information Theory, 2005. Submitted.

[11] J. N. Laneman, D. N. C. Tse, and G. W. Wornell, "Cooperative diversity in wireless networks: Efficient protocols and outage behavior," IEEE Trans. on Information Theory, vol. 50, pp. 3062-3080, Dec. 2004. 
[12] K. Azarian, H. El-Gamal, and P. Schniter, "On the achievable diversity-multiplexing tradeoff in half-duplex cooperative channels," IEEE Trans. on Information Theory, vol. 51, pp. 4152-4172, Dec. 2005.

[13] Y. Oohama, "Coding for relay channels with confidential messages," in Proc. IEEE Information Theory Workshop, (Cairns, Australia), pp. 87 - 89, Sept. 2-7, 2001.

[14] Y. Oohama, "Relay channels with confidential messages," IEEE Trans. on Information Theory, Nov. 2006. Submitted.

[15] I. Csiszar and P. Narayan, "Common randomness and secret key generation with a helper," IEEE Trans. on Information Theory, vol. 24, pp. 339-348, May 1978.

[16] R. Liu, I. Maric, R. D. Yates, and P. Spasojevic, "The discrete memoryless multiple access channel with confidential messages," in Proc. IEEE Internat. Symposium on Information Theory, (Seattle, WA), July 9-14, 2006.

[17] Y. Liang and H. V. Poor, "Generalized multiple access channels with confidential messages," IEEE Trans. on Information Theory, 2006. Submitted.

[18] E. Tekin and A. Yener, "The gaussian multiple access wire-tap channel," IEEE Trans. on Information Theory, 2006. Submitted.

[19] A. O. Hero, "Secure space-time communication," IEEE Trans. on Information Theory, vol. 49, pp. 1-16, Dec. 2003.

[20] C.-M. Zing, F. Kuhlmann, and A. Buzo, "Achivability proof of some multiuser channel coding theorems using backward decoding," IEEE Trans. on Information Theory, vol. 35, no. 6, pp. 1160-1165, 1989.

[21] M. A. Khojastepour, A. Sabharwal, and B. Aazhang, "Cut-set theorems for multi-state networks," IEEE Trans. on Information Theory, 2005. Submitted.

[22] S. K. Leung-Yan-Cheong and M. E. Hellman, "The gaussian wiretap channel," IEEE Trans. on Information Theory, vol. 24, pp. 451-456, Jul. 1978.

[23] T. M. Cover and J. A. Thomas, Elements of Information Theory. New York: Wiley, 1991. 